\title{
BIFURCATION OF CONSTANT MEAN CURVATURE TORI IN EUCLIDEAN SPHERES
}

\author{
LUIS J. ALÍAS AND PAOLO PICCIONE
}

\begin{abstract}
We use equivariant bifurcation theory to show the existence of infinite sequences isometric embeddings of tori with constant mean curvature (CMC) in Euclidean spheres that are not isometrically congruent to the CMC Clifford tori, and accumulating at some CMC Clifford torus.
\end{abstract}

\section{CONTENTS}

1. Introduction

1.1. Index and stability of CMC hypersurfaces

1.2. Bifurcation of CMC Clifford tori

2. An abstract bifurcation setup for equivariant constrained variational problems

2.1. Equivariant constrained variational problems in a Banach setting

2.2. Nondegeneracy and Morse index

2.3. Hilbertization and Fredholmness

2.4. Pseudo-critical points

2.5. The local Palais-Smale condition

2.6. Local Morse invariants

2.7. Equivariant constrained bifurcation

3.2. The area functional

3.3. Volume of a region with boundary $\mathbf{x}(\mathbf{M})$.

3.4. A generalized volume functional

4. Constant mean curvature Clifford tori in the sphere 19

References

\section{INTRODUCTION}

1.1. Index and stability of CMC hypersurfaces. As is well known, minimal hypersurfaces in a Riemannian manifold $N$ are critical points of the variational problem of minimizing area. Similarly, hypersurfaces with constant mean curvature (CMC) in $N$ are also solutions to the same variational problem, when restricted to volume-preserving variations. For such a critical point $M$, the stability equation of the corresponding variational problem is given by the second variation of the area functional, which can be written as the quadratic form $Q(f)=\int_{M}(J f) \cdot f \mathrm{vol}_{M}$ acting on the space of functions on $M$, with

2000 Mathematics Subject Classification. 58J55, 35B32, 53C42.

This work was partially supported by MEC project MTM2009-10418, and Fundación Séneca project 04540/GERM/06, Spain. This research is a result of the activity developed within the framework of the Programme in Support of Excellence Groups of the Región de Murcia, Spain, by Fundación Séneca, Regional Agency for Science and Technology (Regional Plan for Science and Technology 2007-2010).

The second author is partially sponsored by Capes (Brazil), Grant BEX 1509-08-0, and Fundación Séneca grant 09708/IV2/08, Spain. 
$J=-\Delta-m \operatorname{Ric}_{N}(\vec{n})-\|S\|^{2}$. Here $\Delta$ is the Laplacian on functions on $M$ relative to the induced metric, $m=\operatorname{dim}(M)$, $\operatorname{Ric}_{N}(\vec{n})$ is the Ricci curvature of $N$ evaluated on the unit normal field $\vec{n}$ and $S$ is the second fundamental form of the hypersurface. The operator $J$ is called the Jacobi or stability operator of the hypersurface.

In the case of minimal hypersurfaces, the index of a minimal hypersurface $M$, denoted by $\mathfrak{i}_{\text {Morse }}(M)$, is defined as the maximum dimension of any subspace on which $Q$ is negative definite. Equivalently, $\mathfrak{i}_{\text {Morse }}(M)$ is the number of negative eigenvalues of $J$ (counted with multiplicity), which is necessarily finite. Intuitively, $\mathfrak{i}_{\text {Morse }}(M)$ measures the number of independent directions in which the hypersurface fails to minimize area. To see it, observe that if $Q(f)<0$ for a function $f$, then the second derivative of the area functional in the normal variation of $M$ induced by $f$ is negative and therefore $\operatorname{Area}(M)>\operatorname{Area}\left(M_{t}\right)$ for small values of $t$. That means that the minimal hypersurface $M$, while a critical point of the area functional, is not a local minimum. For minimal hypersurfaces in the Euclidean sphere $\mathbb{S}^{m+1}$ this is always the case, and $\mathfrak{i}_{\text {Morse }}(M) \geq 1$ always.

A spectral analysis of the Jacobi differential operator of a given minimal/CMC submanifold provides many information about the displacement of nearby minimal/CMC submanifolds. In [39], Simons characterized the totally geodesic equators $\mathbb{S}^{m} \subset \mathbb{S}^{m+1}$ as the only compact minimal hypersurfaces in $\mathbb{S}^{m+1}$ having $\mathfrak{i}_{\text {Morse }}(M)=1$. Later, Urbano [44], when $m=2$, and El Soufi [20], for general $m$, proved that if $M$ is not a totally geodesic equator, then not only must be $\mathfrak{i}_{\text {Morse }}(M)>1$ but in fact it must hold $\mathfrak{i}_{\text {Morse }}(M) \geq m+3$. On the other hand, apart from the totally geodesic equators, the easiest minimal hypersurfaces in $\mathbb{S}^{m+1}$ are the minimal Clifford tori, and they all have $\mathfrak{i}_{\text {Morse }}(M)=m+3$. For that reason, it has been conjectured for a long time that minimal Clifford tori are the only compact minimal hypersurfaces in $\mathbb{S}^{m+1}$ with $\mathfrak{i}_{\text {Morse }}(M)=m+3$. In [44], Urbano showed that the conjecture is true when $m=2$. Later on, Guadalupe, Brasil Jr. and Delgado [22] showed that the conjecture is true for every dimension $m$, under the additional hypothesis of constant scalar curvature of $M$. More recently, Perdomo [33] proved that the conjecture is also true for every dimension $m$ with an additional assumption about the symmetries of $M$, and, in particular, the conjecture is true for minimal hypersurfaces with antipodal symmetry.

In contrast to the case of minimal hypersurfaces, in the case of hypersurfaces with constant mean curvature one can consider two different eigenvalue problems: the usual Dirichlet problem, associated with the quadratic form $Q$ acting on the whole space of functions on $M$, and the so called twisted Dirichlet problem, associated with the same quadratic form $Q$, but restricted to the subspace of functions $f$ satisfying the additional condition $\int_{M} f \mathrm{vol}_{M}=0$. Similarly, there are two different notions of index, the strong index, denoted by $\mathfrak{i}_{\text {Morse }}^{\mathrm{s}}(M)$ and associated to the usual Dirichlet problem, and the weak index, denoted by $\mathfrak{i}_{\text {Morse }}^{\mathrm{w}}(M)$ and associated to the twisted Dirichlet problem. Specifically, the strong index is simply the maximum dimension of any subspace of functions on which $Q$ is negative definite. On the other hand, the weak index is the maximum dimension of any subspace of functions satisfying $\int_{M} f \mathrm{vol}_{M}=0$ on which $Q$ is negative definite. Obviously, from a geometric point of view the weak index is more natural than the strong index.

Barbosa, do Carmo and Eschenburg [7] characterized the totally umbilical spheres $\mathbb{S}^{m}(r) \subset \mathbb{S}^{m+1}$ as the only compact CMC hypersurfaces in the Euclidean sphere having $\mathfrak{i}_{\text {Morse }}^{\mathrm{w}}(M)=0$ (i.e., being weakly stable). In [3], Alías, Brasil and Perdomo proved that the weak index of any other compact CMC hypersurface $M$ in $\mathbb{S}^{m+1}$ which is not totally umbilical and has constant scalar curvature is greater than or equal to $m+2$, with equality if and only if $M$ is a CMC Clifford torus $\mathbb{S}^{j}(r) \times \mathbb{S}^{m-j}\left(\sqrt{1-r^{2}}\right)$ with radius $\sqrt{j /(m+2)} \leq r \leq \sqrt{(j+2) /(m+2)}$. More recently, in [4] the same authors complemented that estimate by showing that the weak index of any compact CMC hypersurface $M$ in $\mathbb{S}^{m+1}$ which is neither totally umbilical nor a CMC Clifford torus and has constant 
scalar curvature is greater than or equal to $2 m+4$. At this respect, it is worth pointing out that the weak stability index of the CMC Clifford torus $\mathbb{S}^{j}(r) \times \mathbb{S}^{m-j}\left(\sqrt{1-r^{2}}\right)$ depends on $r$ reaching its minimum value $m+2$ when $r \in[\sqrt{j /(m+2)}, \sqrt{(j+2) /(m+2)}]$, and converging to $+\infty$ as $r$ converges either to 0 or 1 (see [3] and Section 4 for the details).

In this paper we study a result of existence for CMC embeddings of the torus $\mathbb{S}^{j} \times \mathbb{S}^{m-j}$ into the sphere $\mathbb{S}^{m+1}$, for arbitrary $1 \leq j<m$. There is a very rich literature on CMC embeddings of the 2-torus $\mathbb{S}^{1} \times \mathbb{S}^{1}$ into $\mathbb{S}^{3}$. Reference [26] contains an extensive description of many recent results of CMC embeddings of cylinders and tori in the 3-sphere, as well as a comprehensive list of references. Two important conjectures are discussed in [26]: the Lawson conjecture and the Pinkall and Sterling conjecture. The Lawson conjecture states that the only embedded minimal 2 -tori in $\mathbb{S}^{3}$ are isometrically congruent to the minimal Clifford torus. The Pinkall and Sterling conjecture states that the only embedded CMC tori in the 3 -sphere are rotational.

A complete classification of rotationally invariant CMC tori in $\mathbb{S}^{3}$ has been given recently in [23]. The authors show the existence of a two-parameter family of CMC surfaces with special spherical symmetry in $\mathbb{S}^{3}$, divided into five types (spheres, Clifford tori ${ }^{1}$, catenoid-type, unduloid-type and nodoid-type), which gives a remarkable analogy with the classical Delaunay classification of rotationally symmetric CMC surfaces in $\mathbb{R}^{3}$.

1.2. Bifurcation of CMC Clifford tori. Let us fix notations in order to give a precise statement of the result proved in this paper. Given a differentiable manifold $M$ and a Riemannian manifold $(N, g)$, we say that two immersions $x_{1}, x_{2}: M \rightarrow N$ of $M$ into $N$ are isometrically congruent when there is an isometry $\psi$ of $(N, g)$ that carries the image of $x_{1}$ onto the image of $x_{2}$, i.e., if there exists a diffeomorphism $\phi$ of $M$ and an isometry $\psi$ of $(N, g)$ such that the following diagram commutes:

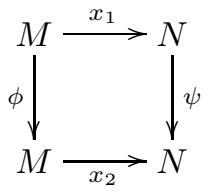

Given integers $m>j \geq 1$ and a positive real number $r \in] 0,1$, we will denote by $x_{r}^{m, j}: \mathbb{S}^{j} \times \mathbb{S}^{m-j} \longrightarrow \mathbb{S}^{m+1}$ the embedding:

$$
x_{r}^{m, j}(p, q)=\left(r \cdot p, \sqrt{1-r^{2}} \cdot q\right), \quad p \in \mathbb{S}^{j}, q \in \mathbb{S}^{m-j} .
$$

These are well known to be embeddings with constant mean curvature (CMC), called in the literature the constant mean curvature Clifford tori. This paper is devoted to proving the following:

Theorem. For fixed $j, m$, there exist two sequences $\left(r_{i}^{m, j}\right)_{i \geq 3}$ and $\left(s_{l}^{m, j}\right)_{l \geq 3}$ in $] 0,1[$, specifically

$$
r_{i}^{m, j}=\sqrt{\frac{(i-2)(j+i-1)}{m-j+(i-2)(j+i-1)}}
$$

and

$$
s_{l}^{m, j}=\sqrt{\frac{j}{j+(l-2)(m-j+l-1)}},
$$

with $\lim _{i \rightarrow \infty} r_{i}^{m, j}=1$ and $\lim _{l \rightarrow \infty} s_{l}^{m, j}=0$, such that the Clifford tori $x_{r_{i}}^{m, j}$ and $x_{s_{l}}^{m, j}$ are accumulation of pairwise non congruent CMC embeddings of $\mathbb{S}^{j} \times \mathbb{S}^{m-j}$ into $\mathbb{S}^{m+1}$, each of which is not congruent to any CMC Clifford torus.

\footnotetext{
${ }^{1}$ Standard tori, in the language of [23]
} 
For all other values of $r \in] 0,1\left[\right.$, the family of CMC Clifford tori $x_{r}^{m, j}$ is locally rigid, in the sense that any CMC embedding of $\mathbb{S}^{j} \times \mathbb{S}^{m-j}$ into $\mathbb{S}^{m+1}$ which is sufficiently close to $x_{r}^{m, j}$ must be isometrically congruent to an embedding of the CMC Clifford family.

It must be observed that, in the case of the 3 -sphere $\mathbb{S}^{3}(j=1$ and $m=2$ in the above statement), our result can be obtained directly from the classification result in [23]; in this case, [23] provides an explicit description of the CMC tori in the bifurcating branches, that are CMC embeddings of unduloid-type.

The basic tool for proving our Theorem is variational bifurcation theory, which requires a quite involved analytical and geometrical framework. A large part of the paper is devoted to establishing the appropriate analytical and geometrical setup for applying bifurcation and Morse theoretical techniques to the CMC variational problem. Let us describe in some detail the technical issues that arise when one tries to give a formal proof of bifurcation of CMC submanifolds.

The problem has a variational nature: $\mathrm{CMC}$ embeddings (in codimension one) are critical points of the area functional defined in the space of embeddings that bound a region of fixed volume. The set of trial maps for the variational problem should be a collection of embeddings of the torus $M=\mathbb{S}^{j} \times \mathbb{S}^{m-j}$ into the sphere $N=\mathbb{S}^{m+1}$; in order to detect solutions that are not isometrically congruent, one should take into consideration the action of the diffeomorphism group of $M$, acting by right composition in the space of embeddings, and the action of the isometry group of $N$, acting by left composition on the space of embeddings. Note that the area and the volume functionals are invariant by the action of these two groups. The action of the diffeomorphism group of $M$ on any set of embeddings of $M$ into $N$ is free, which suggests that one should consider a quotient of the space of embeddings by this action. This means that two embeddings $x, y: M \rightarrow N$ will be considered equivalent if there exists a diffeomorphism $\phi: M \rightarrow M$ such that $y=x \circ \phi$. As to the left action of the isometry group of $N$, this is not free; nevertheless, the group is compact, and one can study a bifurcation problem for its critical orbits. Thus, the variational problem has to be cast in a framework of equivariant bifurcation in a set of equivalence classes of embeddings of $M$ into $N$. One of the crucial issues is the choice of which regularity has to be chosen in the set of embeddings.

In order to apply results from bifurcation theory, the set of trial maps for the variational problem and the functionals involved must satisfy quite restrictive assumptions, more specifically, a (local) Palais-Smale condition, and a Fredholm assumption on the second derivative. The latter is needed for the computation of the local Morse theoretical invariants used to detect bifurcation: roughly speaking, bifurcation occurs at an instant of a path of critical orbits when at this instant there is a jump in the critical groups.

A purely Hilbert structure based on Sobolev spaces seems unfeasible for the type of variational problem treated here. Namely, $H^{1}$-regularity is too loose when the dimension of the source manifold $M$ is greater than one; weak $H^{1}$-solutions of the quasi-linear elliptic equation of $\mathrm{CMC}$ embeddings may fail to be regular, and $H^{1}$-critical points of our variational problem do not give in general CMC embeddings. On the other hand, if one considers Sobolev regularity $H^{k}$, with $k>1$, the second derivative of functionals of the type area/volume is never Fredholm (but rather compact).

The class of embeddings that seems most appropriate for developing a variational theory of the area/volume functional is the set of embeddings of class $C^{2, \alpha}$, with $\left.\alpha \in\right] 0,1[$. However, passing to such a Banach manifold setting has two annoying consequences. First, for the local Palais-Smale condition, which is required in bifurcation theory, it is typically employed a Fredholm assumption for the gradient of the function. Such assumption is satisfied when the second derivative of the function is a Fredholm linear operator from the tangent space to its dual, but typically general Banach spaces (and specifically $C^{2, \alpha}$ ) do not admit any Fredholm operator from the space to its dual. This problem is solved here observing that the second variation of the area functional, which is given by a second order 
linear elliptic operator, although it is not a Fredholm operator from $C^{2, \alpha}$ to its dual, it is represented by a Fredholm operator from $C^{2, \alpha}$ to $C^{0, \alpha}$. The latter space can be put in duality with $C^{2, \alpha}$ using the $L^{2}$-pairing, and in this way one obtains a sort of gradient map which is in fact Fredholm. This yields a local Palais-Smale condition, as in the case of Fredholm maps on Hilbert manifolds.

Second, the notion of nondegeneracy for critical points/orbits suitable for developing local Morse theory in a Banach space context is delicate. Namely, one cannot require that the second variation be an isomorphism from the tangent space to its dual, because again for general Banach spaces there exists no isomorphism from the space to its dual. Recently, a computation of the critical groups for a certain variational problem in Banach spaces has been performed under rather mild nondegeneracy assumtpions (simply, injectivity of the Hessian, see $[9,10])$, but the techniques in $[9,10]$ do not extend in an obvious way to the problem discussed in the present paper. Following [11, 12, 43], the appropriate notion of nondegeneracy for critical points of functions in Banach manifolds has to be given in terms of a sort of splitting of the tangent space into the sum of a strictly positive and a strictly negative subspaces for the Hessian. We will use Chang's notion of s-nondegeneracy for isolated critical points of functionals on Banach manifolds (see [11]), adapted to the case of isolated critical orbits. This is proved here using an extension of the Hessian to the Sobolev space $H^{1}$, in which $C^{2, \alpha}$ sits continuously and densely, together with the fact that, by elliptic regularity, the eigenspaces of such extension are contained in $C^{2, \alpha}$.

These questions are discussed in the first part of the paper, where we present an abstract framework for bifurcation of critical orbits of constrained variational problems on Banach manifolds endowed with a continuous action of a compact Lie group. The framework is described by a set of axioms; such an abstract treatment is not intended with the purpose of developing a general theory, but rather to collect in a unified language the several results from different areas needed for our goal.

Other authors have studied bifurcation of CMC embeddings; for instance, in [32] (see also [36]), it is established a bifurcation result for nodoids, that are immersed (not embedded) CMC cylinders in $\mathbb{R}^{3}$. In the case of nodoids, the hard part of the proof of bifurcation consists in the analysis of the spectrum of the Jacobi operator. The variational setup in that case is quite straightforward, because the ambient space is linear, and so the space of embeddings is an open subset of a Banach space. In our case, the analysis of the spectrum of the Jacobi operator is easier, due to the fact that the Laplacian and the second fundamental form of the CMC Clifford tori in the sphere are well understood objects; for more general CMC tori in the sphere, the spectral analysis of the Jacobi operator is more involved, see $[36,37,38]$. On the other hand, the question of differentiability for the space of embeddings of $M$ into $N$ modulo the diffeomorphisms of $M$ is extremely involved. This issue is studied in detail in Ref. [5]. It should also be observed that the result in [32] is obtained using a classical bifurcation result of Crandall and Rabinowitz, see [15], which allows the authors to obtain also information on the smoothness of the bifurcating branch. The bifurcation result of Crandall and Rabinowitz cannot be employed in the variational problem of the present paper, for the reason that Clifford tori never bifurcate from simple eigenvalues, even working equivariantly. The multiplicity of eigenvalues of CMC Clifford tori is computed in Proposition 4.4. More recently, a bifurcation result of Smoller and Wasserman [41] has been employed to determine bifurcation of fixed boundary nodoids in $\mathbb{R}^{3}$, see [28]. Smoller-Wasserman's deep result gives a sufficient condition for equivariant bifurcation that applies also in situations where there is no jump of the Morse index, in terms of the group representation on the negative eigenspace of the second variation. However, a basic assumption for applying such result is that the original critical branch should consist of fixed points for the group action. This is not the case of the problem studied in this paper, in that CMC Clifford tori are not invariant by the whole isometry group of Euclidean spheres: they have a large stabilizer (see Proposition 4.1), but their 
orbit is not a single point in the space of unparameterized embeddings (Corollary 4.2). It is also worth mentioning the paper [25] by Jost, Li-Jost and Peng, where the authors study a (non equivariant) bifurcation problem for minimal embeddings and relate it to catastrophe theory.

The paper is divided into three parts. In Section 2 we will describe the abstract variational setup that will be employed in the proof of our result. The material is this section deals mostly with Morse theory in Banach manifolds and bifurcation theory, and it is obtained by a systematization of several results already established in the literature in a form suited for our purposes.

In Section 3 we will study the area and volume functionals for arbitrary embeddings of a compact manifold $M$ into a Riemannian manifold $N$, and we will set the CMC embedding variational problem into a Banach manifold framework.

Finally, in Section 4 we will study the specific case of embeddings of $\mathbb{S}^{j} \times \mathbb{S}^{m-j}$ into $\mathbb{S}^{m+1}$. We will show how the abstract bifurcation results apply to the case of CMC Clifford tori, by studying degeneracy, Morse index and stabilizers of the embeddings (1.1), finalizing the proof of our main result.

It should be observed that bifurcation theory does not provide a geometric description of the CMC embeddings that bifurcate from the degenerate CMC Clifford tori.

Acknowledgements. The authors gratefully acknowledge the help provided by Gerard Misiołek (University of Notre Dame, Notre Dame, IN, USA) and Marco Degiovanni (Università Cattolica del Sacro Cuore, Brescia, Italy) during many fruitful conversations. Gerard Misiołek has given several suggestions on the differentiable structure for the set of smooth embeddings of a manifold $M$ into some other manifold $N$ modulo the group of diffeomorphisms of $M$. Marco Degiovanni has helped the authors in the non smooth bifurcation results; among several suggestions, he has pointed out reference [18] that contains the details of a proof of stability of critical groups in the case of critical submanifolds for non smooth functionals on metric spaces.

\section{AN ABSTRACT BIFURCATION SETUP FOR EQUIVARIANT CONSTRAINED VARIATIONAL PROBLEMS}

2.1. Equivariant constrained variational problems in a Banach setting. Let us assume the following setup:

(A1) $\mathfrak{M}$ is a differentiable manifold modeled on a Banach space $X$;

(A2) $G$ is a compact connected Lie group that acts continuously on $\mathfrak{M}$ by homeomorphisms;

(A3) $\mathcal{A}: \mathfrak{M} \rightarrow \mathbb{R}$ is a smooth $G$-invariant function, i.e., $\mathcal{A}(g x)=\mathcal{A}(x)$ for all $g \in G$ and all $x$ in $\mathfrak{M}$.

(A4) $\mathcal{V}: \mathfrak{M} \rightarrow \mathbb{R}$ is a smooth $G$-invariant function without critical points, so that for all $c \in \mathbb{R}$, the inverse image $\Sigma_{c}=\mathcal{V}^{-1}(c)$ is a smooth embedded $G$-invariant submanifold of $\mathfrak{M}$.

For all $x \in \mathfrak{M}$, we will denote by $\mathcal{O}(x, G)$ the $G$-orbit of $x$, and by $G_{x}$ the stabilizer of $x$, which is the closed subgroup of $G$ consisting of all the elements $g$ such that $g x=x$.

We are interested in studying constrained critical points of $\mathcal{A}$ with constraint $\mathcal{V}$, i.e., critical points of the restriction of $\mathcal{A}$ to the hypersurfaces $\Sigma_{c}=\mathcal{V}^{-1}(c)$, when $c$ varies in $\mathbb{R}$. Note that if $x_{0}$ belongs to $\Sigma_{c}$, then the orbit $\mathcal{O}\left(x_{0}, G\right)$ is entirely contained in $\Sigma_{c}$. Moreover, if $x_{0}$ is a critical point of $\left.\mathcal{A}\right|_{\Sigma_{c}}, \mathcal{O}\left(x_{0}, G\right)$ consists entirely of critical points of $\left.\mathcal{A}\right|_{\Sigma_{c}}$. We say then that $\mathcal{O}\left(x_{0}, G\right)$ is a constrained critical orbit of $\mathcal{A}$ subject to the constraint $\mathcal{V}=$ const. We will also set the following axiom:

(A5) for every critical point $x_{0}$ of $\mathcal{A}$ subject to the constraint $\mathcal{V}=$ const, the orbit $\mathcal{O}\left(x_{0}, G\right)$ is a smooth (finite dimensional compact) embedded submanifold of $\mathfrak{M}$, on which the action of $G$ is smooth. 
We observe that, by a result of Dancer [17], assumption (A5) is always satisfied when $\mathfrak{M}$ is a Banach space and the action of $G$ is by linear isomorphisms.

2.2. Nondegeneracy and Morse index. Consider a constrained critical orbit $\mathcal{O}\left(x_{0}, G\right)$ of $\mathcal{A}$ subject to the constraint $\mathcal{V}=$ const. with $\mathcal{V}\left(x_{0}\right)=c$. The tangent space $T_{x_{0}} \mathcal{O}\left(x_{0}, G\right)$ is contained in the kernel of the second variation $\mathrm{d}^{2}\left(\left.\mathcal{A}\right|_{\Sigma_{c}}\right)$; one possible notion of nondegeneracy for the orbit can be given by requiring that the kernel of the bounded symmetric bilinear form $\mathrm{d}^{2}\left(\left.\mathcal{A}\right|_{\Sigma_{c}}\right)\left(x_{0}\right)$ coincides with $T_{x_{0}} \mathcal{O}\left(x_{0}, G\right)$ in $T_{x_{0}} \Sigma_{c}$.

What we will be actually interested in is the question of accumulation of critical orbits corresponding to different values of $c$, and this has to do with another type of degeneracy. For $\lambda \in \mathbb{R}$, define $\mathcal{A}_{\lambda}: \mathfrak{M} \rightarrow \mathbb{R}$ by:

$$
\mathcal{A}_{\lambda}=\mathcal{A}+\lambda \cdot \mathcal{V}
$$

Recall that, by the Lagrange multiplier method, $x_{0} \in \Sigma_{c}$ is a critical point of the restriction $\left.\mathcal{A}\right|_{\Sigma_{c}}$ if and only if there exists a real number $\lambda_{0}$ such that $x_{0}$ is a free critical point of the function $\mathcal{A}_{\lambda_{0}}$ (note that $\lambda_{0}$ for all the critical points of the orbit $\mathcal{O}\left(x_{0}, G\right)$ ). Moreover, in this case the second variation of $\left.\mathcal{A}\right|_{\Sigma_{c}}$ at $x_{0}$ is given by the restriction of the second variation $\mathrm{d}^{2} \mathcal{A}_{\lambda_{0}}\left(x_{0}\right)$ to $T_{x_{0}} \Sigma_{c}$. We will say that $\mathcal{O}\left(x_{0}, G\right)$ is a nondegenerate constrained critical orbit of $\mathcal{A}$ subject to the constraint $\mathcal{V}=$ const. if the kernel of the symmetric bilinear form $\mathrm{d}^{2} \mathcal{A}_{\lambda_{0}}\left(x_{0}\right)$ in $T_{x_{0}} \mathfrak{M}$ is equal to $T_{x_{0}} \mathcal{O}\left(x_{0}, G\right)$. Note that this is a weaker notion of nondegeneracy compared to the classical nondegeneracy of critical points for functions on a Hilbert manifold, where one requires that the second derivative $\mathrm{d}^{2} \mathcal{A}_{\lambda_{0}}\left(x_{0}\right)$ be an isomorphism from the tangent space $T_{x_{0}} \mathfrak{M}$ to its dual, and not just an injective map. In the classical Hilbert setting, injectivity of the second derivative is not a condition strong enough to develop Morse theory. Appropriate Fredholmness assumptions will be imposed later, which will imply that our notion of nondegeneracy is suitable for applying Morse theoretical techniques.

Similarly, there are two distinct notions of Morse index of a constrained critical orbits. Given a (nondegenerate) constrained critical orbit $\mathcal{O}\left(x_{0}, G\right)$ with $\mathcal{V}\left(x_{0}\right)=c$ and with Lagrange multiplier $\lambda_{0}$, we define the weak Morse index $\mathfrak{i}_{\text {Morse }}^{\mathrm{w}}\left(\mathcal{O}\left(x_{0}, G\right)\right)$ the (possibly infinite) dimension of a maximal subspace of $T_{x_{0}} \Sigma_{c}$ on which $\mathrm{d}^{2} \mathcal{A}_{\lambda_{0}}\left(x_{0}\right)$ is negative definite. By the strong Morse index $\mathfrak{i}_{\text {Morse }}^{\mathrm{s}}\left(\mathcal{O}\left(x_{0}, G\right)\right)$ we mean the dimension of a maximal subspace of $T_{x_{0}} \mathfrak{M}$ on which $\mathrm{d}^{2} \mathcal{A}_{\lambda_{0}}\left(x_{0}\right)$ is negative definite. Since $\Sigma_{c}$ has codimension 1 in $\mathfrak{M}$, it follows immediately that either both the strong and the weak Morse index are infinite, or the following inequalities hold:

$$
\mathfrak{i}_{\text {Morse }}^{\mathrm{w}}\left(\mathcal{O}\left(x_{0}, G\right)\right) \leq \mathfrak{i}_{\text {Morse }}^{\mathrm{s}}\left(\mathcal{O}\left(x_{0}, G\right)\right) \leq \mathfrak{i}_{\text {Morse }}^{\mathrm{w}}\left(\mathcal{O}\left(x_{0}, G\right)\right)+1 .
$$

2.3. Hilbertization and Fredholmness. Although the basic framework for the variational problems considered in this paper is given by manifolds modeled on Banach spaces, it will be important to have an underlying Hilbert structure, on which the second derivative of our functionals must be represented by self-adjoint Fredholm operators. In the purely Banach space context, the assumption of Fredholmness for the second derivative of functionals is not reasonable, as in most cases there exists no Fredholm operator between a Banach space and its dual.

A Hilbert/Fredholm structure will be employed in this paper in three different ways. First, Fredholmness of the second derivative is used to prove a local compactness condition (Palais-Smale condition) for our functionals. Second, for the proof of rigidity of the Clifford family away from the jumps of the Morse index we will need to use a version of the implicit function theorem which requires Fredholmness. Third, a Hilbert/Fredholm structure is needed in order to employ Morse theoretical techniques in the Banach manifold context, in the spirit of $[11,43]$.

Two different sets of axioms are required. Axiom (HF-A) deals with the notion of gradient map for the functionals $\mathcal{A}_{\lambda}$, and it has the main purpose to guarantee a local (PS) 
condition (see Proposition 2.2). Axiom (HF-B) deals with the second derivative of $\mathcal{A}_{\lambda}$, and this is related to the computation of the local homological invariants of Morse theory in the Banach manifold setting. It will be used essentially in Proposition 2.3. The reader may keep in mind that, in our applications, the Banach space $X$ is given by the space of $C^{k, \alpha}$ sections $(k \geq 2)$ of some Riemannian vector bundle $E$ over a compact manifold $M$, the Banach space $Y$ is the space of $C^{k-2, \alpha}$ sections of $E, \mathcal{H}_{0}$ is the space of $L^{2}$-sections of $E$ and $\mathcal{H}_{1}$ is the space of sections of $E$ having Sobolev class $H^{1}$.

(HF-A) Given a critical point $x_{0}$ of $\mathcal{A}_{\lambda_{0}}$, there exists an open neighborhood $U$ of $x_{0}$ diffeomorphic to an open subset of the Banach space $X$ (the model of $\mathfrak{M}$ ), another Banach space $Y$ and a Hilbert space $\left(\mathcal{H}_{0},\langle\cdot, \cdot\rangle_{0}\right)$, with continuous inclusions $X \hookrightarrow Y \hookrightarrow \mathcal{H}_{0}$ having dense images, and a map

$$
H:] \lambda_{0}-\varepsilon, \lambda_{0}+\varepsilon[\times U \longrightarrow Y
$$

of class $C^{1}$ such that:

$$
\mathrm{d} \mathcal{A}_{\lambda}(x) v=\langle H(\lambda, x), v\rangle_{0},
$$

for all $x \in U, \lambda \in] \lambda_{0}-\varepsilon, \lambda_{0}+\varepsilon[$ and $v \in X$, whose partial derivative:

$$
\frac{\partial H}{\partial x}\left(\lambda_{0}, x_{0}\right): X \longrightarrow Y
$$

is a Fredholm linear map of index 0 . We will call such $H$ a gradient map for the family $\mathcal{A}_{\lambda}$.

(HF-B) Given a critical point $x_{0}$ of $\mathcal{A}_{\lambda_{0}}$, there exists a Hilbert space $\mathcal{H}_{1}$, a continuous inclusion $X \hookrightarrow \mathcal{H}_{1}$ having dense image, an open neighborhood $U$ of $x_{0}$ in $\mathfrak{M}$ diffeomorphic to an open subset $V$ of $X$, such that (identifying $U$ and $V$ with such diffeomorphism and considering $\mathcal{A}_{\lambda_{0}}$ as a function on $V$ ):

(HF-B1) for $x \in V$, the second derivative $\mathrm{d}^{2} \mathcal{A}_{\lambda_{0}}(x)$ admits an extension to a bounded essentially positive symmetric bilinear form on $\mathcal{H}_{1}$, represented by the (selfadjoint) operator $S_{\lambda_{0}, x}$ on $\mathcal{H}_{1}$;

(HF-B2) there exists $\delta>0$ such that, for $\sigma \in]-\infty, \delta]$, the $\sigma$-eigenspace of the essentially positive operator $S_{\lambda_{0}, x_{0}}$ is contained in $T_{x_{0}} \mathfrak{M}$.

We will also require that the above objects depend continuously on $x_{0}$ and $\lambda_{0}$ :

(HF-B3) Axioms (HF-B1) and (HF-B2) hold for every $\lambda$ near $\lambda_{0}$, and the map $S_{\lambda, x}$ depend continuously on $\lambda$ and $x$.

By an essentially positive self-adjoint operator on a Hilbert space we mean an operator of the form $P+K$, where $P$ is a positive isomorphism and $K$ is a self-adjoint compact operator. Equivalently, essentially positive operators are self-adjoint Fredholm operators whose essential spectrum in contained in $] 0,+\infty[$. If $S$ is an essentially positive selfadjoint operator, then there exists $\delta>0$ such that, if $\mathfrak{s}(S) \subset \mathbb{R}$ denotes the spectrum of $S$, the intersection $\mathfrak{s}(S) \cap]-\infty, \delta]$ consists of a finite number of eigenvalues, each of which has finite multiplicity. A symmetric bilinear form $B$ on a Hilbert space is called essentially positive if $B=\langle S \cdot, \cdot\rangle$ for some essentially positive operator $S$. This notion is independent on the choice of a Hilbert space inner product $\langle\cdot, \cdot\rangle$ on $H$.

A few comments on the HF-axioms are in order. First, we observe that the assumption on the density of the inclusions $X \hookrightarrow Y \hookrightarrow \mathcal{H}_{0}$ in (HF-A) implies that $\mathrm{d} \mathcal{A}_{\lambda}(x)=0$ if and only if the gradient $H(\lambda, x)$ vanishes. Namely, if $\mathrm{d} \mathcal{A}_{\lambda}(x)=0$, then $H(\lambda, x)$ is orthogonal to the dense subspace $X$.

The second observation is that the strong nondegeneracy for a critical orbit $\mathcal{O}\left(x_{0}, G\right)$ is equivalent to the fact that the kernel of the derivative $\frac{\partial H}{\partial x}\left(\lambda_{0}, x_{0}\right)$ has dimension equal to the dimension of $\mathcal{O}\left(x_{0}, G\right)$. By the Fredholmness assumtpion, this implies that, given any closed complement $X_{2}$ of $T_{x_{0}} \mathcal{O}\left(x_{0}, G\right)$ in $X$, the linear map $\frac{\partial H}{\partial x}\left(\lambda_{0}, x_{0}\right)$ restricts to a Banach space isomorphism between $X_{2}$ and the image of $\frac{\partial H}{\partial x}\left(\lambda_{0}, x_{0}\right)$. 
Third, assumption (HF-B2) implies that $\mathcal{O}\left(x_{0}, G\right)$ is a nondegenerate critical orbit for $\mathcal{A}_{\lambda_{0}}$ if the self-adjoint operator $S_{\lambda_{0}, x_{0}}$ is an isomorphism of $\mathcal{H}_{1}$. Moreover, (HF-B1) and (HF-B2) imply that the strong Morse index $\mathfrak{i}_{\text {Morse }}^{\text {s }}\left(\mathcal{O}\left(x_{0}, G\right)\right)$ is finite and equal to the sum of the dimensions of the negative eigenspaces of $S_{\lambda_{0}, x_{0}}$.

Finally, it should be remarked that in specific examples the Hilbert spaces $\mathcal{H}_{0}$ and $\mathcal{H}_{1}$ in Axioms (HF-A) and (HF-B) may be related. For instance, in the situation described above where $X$ is the Banach space of $C^{2, \alpha}$-sections of a Riemannian vector bundle $E$ over a compact manifold $M$, if the derivative $\frac{\partial H}{\partial x}\left(\lambda_{0}, x_{0}\right)$ is an elliptic operator, then the Hilbert space $\mathcal{H}_{1}$ can be defined as the Hilbert space completion of $X$ with respect to the inner product

$$
\left\langle x_{1}, x_{2}\right\rangle_{1}=\left\langle x_{1}, x_{2}\right\rangle_{0}+\left\langle\frac{\partial H}{\partial x}\left(\lambda_{0}, x_{0}\right) x_{1}, x_{2}\right\rangle_{0} .
$$

2.4. Pseudo-critical points. Let us use the axiom (HF-A) to show a preliminary result on the distribution of critical points of the family $\mathcal{A}_{\lambda}$. In the variational setup (A1)-(A5), assume that (HF-A) holds in a neighborhood $U$ critical point $x_{0}$ of $\mathcal{A}_{\lambda_{0}}$. Set:

$$
X_{1}=\operatorname{Ker}\left[\frac{\partial H}{\partial x}\left(\lambda_{0}, x_{0}\right)\right], \quad Y_{2}=\operatorname{Im}\left[\frac{\partial H}{\partial x}\left(\lambda_{0}, x_{0}\right)\right] ;
$$

let $X_{2}$ be a closed complement of $X_{1}$ in $X$ and $Y_{1}$ be a closed complement of $Y_{2}$ in $Y$. Note that $\operatorname{dim}\left(X_{1}\right)=\operatorname{dim}\left(Y_{1}\right)=d$, by the zero index assumption on the Fredholm map $\frac{\partial H}{\partial x}\left(\lambda_{0}, x_{0}\right)$. Let $P_{2}: Y \rightarrow Y_{2}$ be the projection relative to the direct sum decomposition $Y=Y_{1} \oplus Y_{2}$, and set $H_{2}=P_{2} \circ H$. A point $(\bar{\lambda}, \bar{x})$ in the domain of $H$ will be called a pseudo-critical point for the family $\mathcal{A}_{\lambda}$ if $H_{2}(\bar{\lambda}, \bar{x})=0$. Under certain assumptions (see Proposition 2.4), we will show that pseudo-critical points are in fact critical. Next Lemma tells us how pseudo-critical points are displaced near a nondegenerate critical orbit.

Lemma 2.1. In the variational setup $(A 1)-(A 5)$, let $x_{0} \in \mathfrak{M}$ be a critical point of $\mathcal{A}_{\lambda_{0}}$ whose critical orbit $\mathcal{O}\left(x_{0}, G\right)$ has dimension $d$ and is nondegenerate. Assume that $(H F-A)$ holds at $x_{0}$. Then, there exists an open neighborhood $W$ of $\left(\lambda_{0}, x_{0}\right)$ in $\mathbb{R} \times \mathfrak{M}$ and $a$ $(d+1)$-dimensional submanifold $\mathcal{D} \subset W$ such that, for $(\bar{\lambda}, \bar{x}) \in W,(\bar{\lambda}, \bar{x})$ is a pseudocritical point for the family $\mathcal{A}_{\lambda}$ if and only if $(\bar{\lambda}, \bar{x}) \in \mathcal{D}$.

Proof. Let $a_{0} \in X_{1}$ and $b_{0} \in X_{2}$ be such that $x_{0}=a_{0}+b_{0}$. Consider the $C^{1}$-map $\mathcal{F}$, defined in a neighborhood of $\left(\lambda_{0}, a_{0}, b_{0}\right)$ in $\mathbb{R} \times X_{1} \times X_{2}$ and taking values in a neighborhood of $\left(\lambda_{0}, a_{0}, 0\right)$ in $\mathbb{R} \times X_{1} \times Y_{2}$, obtained by setting:

$$
\mathcal{F}(\lambda, a, b)=\left(\lambda, a, H_{2}(\lambda, a+b)\right) .
$$

We claim that $\mathcal{F}$ is a diffeomorphism around the point $\left(\lambda_{0}, a_{0}, b_{0}\right)$; in order to prove the claim it suffices to apply the Inverse Mapping Theorem, observing that the differential $\mathrm{d} \mathcal{F}\left(\lambda_{0}, a_{0}, 0\right)$ is written in block form as:

$$
\mathrm{d} \mathcal{F}\left(\lambda_{0}, a_{0}, 0\right)=\left(\begin{array}{ccc}
1 & 0 & 0 \\
0 & \mathrm{Id} & 0 \\
* & * & T
\end{array}\right)
$$

where $T=\frac{\partial H_{2}}{\partial b}\left(\lambda_{0}, a_{0}, b_{0}\right): X_{2} \rightarrow Y_{2}$, and Id is the identity map of $X_{1}$. Now, $\mathrm{d} \mathcal{F}\left(\lambda_{0}, a_{0}, 0\right)$ is an isomorphism, as $T$ is an isomorphism. Notice in fact that $T$ is the restriction of $\frac{\partial H}{\partial x}\left(\lambda_{0}, x_{0}\right)$ to $X_{1}$. Then, the set of pseudo-critical points for the family $\mathcal{A}_{\lambda}$ in a small neighborhood of $\left(\lambda_{0}, a_{0}, b_{0}\right)$ is the graph of the $C^{1}$-function $\psi$, defined in a neighborhood of $\left(\lambda_{0}, a_{0}\right)$ in $\mathbb{R} \times X_{1}$ and taking values in a neighborhood of $b_{0}$ in $X_{2}$, defined by $\psi(\lambda, a)=\pi\left(\mathcal{F}^{-1}(\lambda, a, 0)\right)$, where $\pi: \mathbb{R} \times X_{1} \times X_{2} \rightarrow X_{2}$ is the projection onto the third coordinate. The graph of $\psi$ is a $(d+1)$-dimensional $C^{1}$-submanifold of $\mathbb{R} \times X$, which is identified with a $(d+1)$-dimensional $C^{1}$-submanifold $\mathcal{D}$ of $\mathbb{R} \times \mathfrak{M}$. 
2.5. The local Palais-Smale condition. Recall that a Palais-Smale sequence for the functional $\mathcal{A}_{\lambda}$ is a sequence $\left(x_{n}\right)_{n \in \mathbb{N}}$ such that $\left|\mathcal{A}_{\lambda}\left(x_{n}\right)\right|$ is bounded and $\left\|\mathrm{d} \mathcal{A}\left(x_{n}\right)\right\|$ is infinitesimal. Given a (closed) subset $\mathfrak{C} \subset \mathfrak{M}$, the functional $\mathcal{A}_{\lambda}$ is said to satisfy the Palais-Smale condition in $\mathfrak{C}$ if every Palais-Smale sequence for $\mathcal{A}_{\lambda}$ contained in $\mathfrak{C}$ has a converging subsequence.

An adaptation of a classical result of Marino and Prodi (see [31]), gives the following:

Proposition 2.2. In the variational setup described by (AI)-(A5), assume that (HF-A) is satisfied at every point of a constrained critical orbit $\mathcal{O}\left(x_{0}, G\right)$ of the functional $\mathcal{A}_{\lambda_{0}}$. Then, given $\varepsilon>0$ sufficiently small, there exists a closed neighborhood $W$ of $\mathcal{O}\left(x_{0}, G\right)$ such that, for all $\lambda \in] \lambda_{0}-\varepsilon, \lambda_{0}+\varepsilon\left[, \mathcal{A}_{\lambda}\right.$ satisfies the Palais-Smale condition in $W$.

Proof. Using the compactness of the orbit and the $G$-equivariance, it suffices to show the existence of a closed neighborhood of $x_{0}$ on which $\mathcal{A}_{\lambda}$ satisfies the Palais-Smale condition for all $\lambda$ sufficiently close to $\lambda_{0}$. Since the set of Fredholm operators is open in the space of all bounded operators from $X$ to $Y$ and the map $H$ is of class $C^{1}$, by taking $\varepsilon>0$ and $U$ sufficiently small we can assume that the partial derivative $\frac{\partial H}{\partial x}(\lambda, z)$ is Fredholm for all $z \in$ $U$. The local form of a $C^{1}$-map between Banach spaces with Fredholm derivative (see for instance [1, Theorem 1.7, p. 4]) says that there is a $C^{1}$-change of coordinates that carries a neighborhood of $x_{0}$ in $U$ to a neighborhood of zero of a Banach space direct sum $E_{1}^{\lambda} \oplus E_{2}^{\lambda}$, with $\operatorname{dim}\left(E_{2}^{\lambda}\right)<+\infty$, and that takes $x_{0}$ to $(0,0)$, and a $C^{1}$-change of coordinates that carries a neighborhood of $H\left(\lambda_{0}, x_{0}\right)$ in $Y$ to a neighborhood of zero of another Banach space direct sum $F_{1}^{\lambda} \oplus E_{1}^{\lambda}$ with $\operatorname{dim}\left(F_{1}^{\lambda}\right)<+\infty$, and that takes $H\left(\lambda_{0}, x_{0}\right)$ to $(0,0)$, such that, using these coordinates, the map $H(\lambda, \cdot)$ takes the form $E_{1}^{\lambda} \oplus E_{2}^{\lambda} \ni(u, v) \mapsto$ $\left(\eta_{\lambda}(u, v), u\right) \in F_{1}^{\lambda} \oplus E_{1}^{\lambda}$, where $\eta_{\lambda}: E_{1}^{\lambda} \oplus E_{2}^{\lambda} \rightarrow F_{1}^{\lambda}$ a $C^{1}$-map with d $\eta_{\lambda}(0,0)=0$. It is immediate so see that such a map is proper when restricted to the unit ball of $E_{1}^{\lambda} \oplus E_{2}^{\lambda}$, thus the map $H(\lambda, \cdot)$ is proper when restricted to a suitable closed neighborhood $W^{\lambda}$ of $x_{0}$, depending on $\lambda$. The size of $W^{\lambda}$ depends continuously on $H$ and $\frac{\partial H}{\partial x}$; a proof of this assertion is obtained easily by keeping track of sizes in the proof of [1, Theorem 1.7, p. 4], that uses the inverse function theorem. Thus, using the continuity of $H$ and $\frac{\partial H}{\partial x}$, one can find a fixed neighborhood $W_{0}$ of $x_{0}$ such that for all $\lambda$ sufficiently close to $\lambda_{0}, H(\lambda, \cdot)$ is proper when restricted to $W_{0}$.

Given a Palais-Smale sequence $\left(x_{n}\right)_{n \in \mathbb{N}}$ for $\mathcal{A}_{\lambda}$ contained in such neighborhood, then $H\left(\lambda, x_{n}\right)$ tends to 0 as $n \rightarrow \infty$, and thus the set $K=\left\{H\left(\lambda, x_{n}\right): n \in \mathbb{N}\right\} \bigcup\{0\}$ is compact. The sequence $\left(x_{n}\right)_{n \in \mathbb{N}}$ is contained in the compact subset $H(\lambda, \cdot)^{-1}(K)$, and therefore it admits a converging subsequence, which concludes the proof.

2.6. Local Morse invariants. Let $x_{0}$ be a critical point of $\mathcal{A}_{\lambda_{0}}$, and let $\mathcal{O}=\mathcal{O}\left(x_{0}, G\right)$ be the critical orbit of $x_{0}$; set $c=\mathcal{A}_{\lambda_{0}}\left(x_{0}\right)$. For $q \in \mathbb{R}$, define $\mathcal{A}_{\lambda_{0}}^{q}$ the closed sublevel:

$$
\mathcal{A}_{\lambda_{0}}^{q}=\left\{x \in \mathfrak{M}: \mathcal{A}_{\lambda_{0}}(x) \leq q\right\} .
$$

Given a coefficient ring $\mathbb{F}$, the sequence of $\mathbb{F}$-critical groups of $\mathcal{O}$ is the sequence

$$
\mathfrak{H}_{*}\left(\mathcal{O}, \lambda_{0} ; \mathbb{F}\right)=\left(\mathfrak{H}_{\nu}\left(\mathcal{O}, \lambda_{0} ; \mathbb{F}\right)\right)_{\nu \in \mathbb{N}}
$$

of relative (singular) homology groups with coefficients in $\mathbb{F}$ :

$$
\mathfrak{H}_{\nu}\left(\mathcal{O}, \lambda_{0} ; \mathbb{F}\right)=H_{\nu}\left(\mathcal{A}_{\lambda_{0}}^{c}, \mathcal{A}_{\lambda_{0}}^{c} \backslash \mathcal{O} ; \mathbb{F}\right)
$$

By excision, if $U$ is any open set of $\mathfrak{M}$ that contains $\mathcal{O}$, then:

$$
\mathfrak{H}_{\nu}\left(\mathcal{O}, \lambda_{0} ; \mathbb{F}\right)=H_{\nu}\left(\mathcal{A}_{\lambda_{0}}^{c} \cap U,\left(\mathcal{A}_{\lambda_{0}}^{c} \cap U\right) \backslash \mathcal{O} ; \mathbb{F}\right)
$$

for all $\nu \in \mathbb{N}$.

We will now show how are assumptions can be used to compute the critical groups of a nondegenerate orbit. Such computation is based on an analysis of appropriate $G$-invariant neighborhoods of a critical orbit; it will be useful to employ some terminology from principal fiber bundles. Recall that given an $H$-principal bundle $P \rightarrow X$ over the manifold $X$, 
and given a topological space $Y$ endowed with an $H$-action, the twisted product $P \times_{H} Y$ is a fiber bundle over $X$ whose fiber at $x \in X$ in the quotient of the product $P_{x} \times Y$ by the left action of $H$ given by:

$$
H \times\left(P_{x} \times Y\right) \ni(h,(p, y)) \mapsto\left(p h^{-1}, h y\right) \in P_{x} \times Y .
$$

Since the right action of $H$ on $P_{x}$ is free and transitive, such quotient is homeomorphic to $Y ; P \times_{Y} X$ is fiber bundle over $X$ with typical fiber $Y$. When $G$ is a compact Lie group acting on a completely regular topological space $X$, then through every $x \in X$ there is a slice $\Sigma_{x}$ (see [8, Chapter II, Sec. 5]); recall that $\Sigma_{x}$ is a subset of $X$, which is invariant by the action of the stabilizer $H$ of $x$, and such that the map $G \times \Sigma_{x} \ni(g, y) \mapsto g \cdot y \in X$ defines an homeomorphism of the twisted product $G \times{ }_{H} \Sigma_{x}$ and an open neighborhood of the orbit $G x$.

For the computation of the critical groups in our setup, we will consider for simplicity the field $\mathbb{F}=\mathbb{Z}_{2}$.

Proposition 2.3. In the variational setup (A1)-(A5), let $x_{0} \in \mathfrak{M}$ be a critical point of $\mathcal{A}_{\lambda_{0}}$. Assume that $(H F-B)$ holds around the points of the critical orbit $\mathcal{O}=\mathcal{O}\left(x_{0}, G\right)$, that $(H F-A)$ holds at every point of $\mathcal{O}$ and that $\mathcal{O}\left(x_{0}, G\right)$ is nondegenerate. Set $\mu=$ $\mathfrak{i}_{\text {Morse }}^{\mathrm{s}}\left(\mathcal{O}\left(x_{0}, G\right)\right)$. Then for all $\nu \in \mathbb{N}$, the critical group $\mathfrak{H}_{\nu}\left(\mathcal{O} ; \mathbb{Z}_{2}\right)$ is isomorphic to the singular homology group $H_{\nu-\mu}\left(\mathcal{O} ; \mathbb{Z}_{2}\right)$ of the orbit $\mathcal{O}$.

Proof. Let $H$ be the stabilizer of $x_{0}$, and consider the principal fiber bundle $G \longrightarrow G / H \cong$ $\mathcal{O}\left(x_{0}, G\right)$. Let $\Sigma_{x_{0}}$ be a slice at $x_{0}$, let $U$ be the open neighborhood of $\mathcal{O}$ given by $G \cdot \Sigma_{x_{0}}$ and set $c=\mathcal{A}_{\lambda_{0}}\left(x_{0}\right)$. The intersection $\mathcal{A}_{\lambda_{0}}^{c} \cap U$ is a fiber bundle over $\mathcal{O}$, whose typical fiber is the intersection $\Sigma_{x_{0}}^{c}=\mathcal{A}_{\lambda_{0}}^{c} \cap \Sigma_{x_{0}}$. Let $\mathcal{S}$ be a submanifold of $\mathfrak{M}$ through $x_{0}$ which is transversal to $\mathcal{O}$ at $x_{0}$ (i.e., $T_{x_{0}} \mathcal{S} \cap T_{x_{0}} \mathcal{O}=\{0\}$ and $T_{x_{0}} \mathcal{S}+T_{x_{0}} \mathcal{O}=T_{x_{0}} \mathfrak{M}$ ); consider a homeomorphism $\mathfrak{h}$ from $\Sigma_{x_{0}}$ to $\mathcal{S}$, with $\mathfrak{h}\left(x_{0}\right)=x_{0}$, and such that $\mathcal{A}_{\lambda_{0}} \circ \mathfrak{h}=\mathcal{A}_{\lambda_{0}}$ on $\Sigma_{x_{0}}$. Then, $\mathfrak{h}$ carries $\mathcal{A}_{\lambda_{0}}^{c} \cap \Sigma_{x_{0}}$ to $\mathcal{A}_{\lambda_{0}}^{c} \cap \mathcal{S}$ and $\left(\mathcal{A}_{\lambda_{0}}^{c} \cap \Sigma_{x_{0}}\right) \backslash\left\{x_{0}\right\}$ to $\mathcal{A}_{\lambda_{0}}^{c} \cap \mathcal{S} \backslash\left\{x_{0}\right\}$, thus:

$$
H_{k}\left(\left(\mathcal{A}_{\lambda_{0}}^{c} \cap \Sigma_{x_{0}}\right),\left(\mathcal{A}_{\lambda_{0}}^{c} \cap \Sigma_{x_{0}}\right) \backslash\left\{x_{0}\right\} ; \mathbb{Z}_{2}\right) \cong H_{k}\left(\left(\mathcal{A}_{\lambda_{0}}^{c} \cap \mathcal{S}\right),\left(\mathcal{A}_{\lambda_{0}}^{c} \cap \mathcal{S}\right) \backslash\left\{x_{0}\right\} ; \mathbb{Z}_{2}\right)
$$

for all $k$. The restriction of $\mathcal{A}_{\lambda_{0}}$ to $\mathcal{S}$ is a smooth function, and it has $x_{0}$ as an isolated critical point. This follows easily from the fact that, by the $G$-invariance, the critical points of the restriction of $\mathcal{A}_{\lambda_{0}}$ to $\mathcal{S}$ are precisely the critical points of $\mathcal{A}_{\lambda_{0}}$ that lie on $\mathcal{S}$. Then, since $\mathcal{O}\left(x_{0}, G\right)$ is nondegenerate, it is an isolated critical orbit of $\mathcal{A}_{\lambda_{0}}$, which has an isolated intersection with $\mathcal{S}$ at $x_{0}$, by transversality.

Now, the critical point $x_{0}$ of the restriction of $\mathcal{A}_{\lambda_{0}}$ to $\mathcal{S}$ is s-nondegenerate, in the sense of [11]. Recall that this means that there exists a diffeomorphism from an open subset $\mathcal{V}$ of the Banach space $T_{x_{0}} \mathcal{S}$ to an open neighborhood of $x_{0}$ in $\mathcal{S}$ and a hyperbolic isomorphism $L: T_{x_{0}} \mathcal{S} \rightarrow T_{x_{0}} \mathcal{S}$ such that, using such diffeomorphism to identify $\mathcal{A}_{\lambda_{0}}$ with a smooth function on $\mathcal{V}$, the following conditions are satisfied:

(a) $\mathrm{d}^{2} \mathcal{A}_{\lambda_{0}}\left(x_{0}\right)[L v, w]=\mathrm{d}^{2} \mathcal{A}_{\lambda_{0}}\left(x_{0}\right)[v, L w]$ for all $v, w \in T_{x_{0}} \mathcal{S}$;

(b) $\mathrm{d}^{2} \mathcal{A}_{\lambda_{0}}\left(x_{0}\right)[L v, v]>0$ for all $v \in T_{x_{0}} \mathcal{S}, v \neq 0$;

(c) $\mathrm{d} \mathcal{A}_{\lambda_{0}}(x)\left[L\left(x-x_{0}\right)\right]>0$ for all $x$ in $\mathcal{V} \backslash\left\{x_{0}\right\}$ with $\mathcal{A}_{\lambda_{0}}(x) \leq \mathcal{A}_{\lambda_{0}}\left(x_{0}\right)$.

We will use Axioms (HF-B) to verify s-nondegeneracy. Consider the open subset $V$ of the Banach space $X \cong T_{x_{0}} \mathfrak{M}$, the Hilbert space $\mathcal{H}_{1}$ and the essentially positive operator $S_{x_{0}, \lambda_{0}}$ on $\mathcal{H}_{1}$ as in (HF-B). Denote by $T_{x_{0}} \mathcal{O}^{\perp}$ the orthogonal complement of $T_{x_{0}} \mathcal{O}$ in $\mathcal{H}_{1}$. Then, setting $\bar{X}=X \cap T_{x_{0}} \mathcal{O}^{\perp}$, one has $X=T_{x_{0}} \mathcal{O} \oplus \bar{X}$, because $T_{x_{0}} \mathcal{O} \subset X$; moreover $\bar{X}$ is isomorphic to $T_{x_{0}} \mathcal{S}$. Choose a local chart of $\mathfrak{M}$ around $x_{0}$ taking values in $T_{x_{0}} \mathcal{O} \oplus \bar{X}$ and carrying an open neighborhood of $x_{0}$ in $\mathcal{S}$ to an open neighborhood of 0 in $\{0\} \oplus \bar{X} \cong \bar{X} \cong T_{x_{0}} \mathcal{S}$.

The Hilbert space $T_{x_{0}} \mathcal{O}^{\perp}$ is invariant by $S_{\lambda_{0}, x_{0}}$, and it splits as an orthogonal direct sum $\mathcal{H}_{-} \oplus \mathcal{H}_{*} \oplus \mathcal{H}_{+}$, where $\mathcal{H}_{-}$is finite dimensional and it is spanned by the eigenvectors of 
$S_{\lambda_{0}, x_{0}}$ having negative eigenvalue, $\mathcal{H}_{*}$ is finite dimensional and it is spanned by the eigenvectors of $S_{\lambda_{0}, x_{0}}$ having eigenvalue in $\left.] 0, \delta\right]$, and $\mathcal{H}_{+}$has infinite dimension, is invariant by $S_{\lambda_{0}, x_{0}}$ and the restriction of $S_{\lambda_{0}, x_{0}}$ to $\mathcal{H}_{+}$has spectrum contained in $] \delta,+\infty$ [. By assumption (HB-3), $\mathcal{H}_{-}$and $\mathcal{H}_{*}$ are contained in $\bar{X}$, and thus $\bar{X}=\mathcal{H}_{-} \oplus \mathcal{H}_{*} \oplus\left(\bar{X} \cap \mathcal{H}_{+}\right)$. Let $L: \bar{X} \rightarrow \bar{X}$ be the isomorphism whose restriction to $\mathcal{H}_{-}$is minus the identity, and whose restriction to $\mathcal{H}_{*} \oplus\left(\bar{X} \cap \mathcal{H}_{+}\right)$is the identity. Evidently, $L$ is hyperbolic. Such operator admits an extension to a self-adjoint isomorphism of $\mathcal{H}_{1}$, and properties (a) and (b) above are readily verified for such extension. Namely, it is easy to see that $L$ commutes with $S_{\lambda_{0}, x_{0}}$, which implies that (a) holds. Property (b) is obvious, using the fact that the spaces $\mathcal{H}_{-}$and $\mathcal{H}_{*} \oplus\left(\bar{X} \cap \mathcal{H}_{+}\right)$are orthogonal and invariant by $S_{\lambda_{0}, x_{0}}$; the composition $S_{\lambda_{0}, x_{0}} L$ is a positive isomorphism of $\mathcal{H}_{1}$. Property (c) is also obtained easily using the mean value theorem for the function $t \mapsto \mathrm{d} \mathcal{A}_{\lambda_{0}}\left(x_{0}+t v\right)[L v]$, where $v=x-x_{0}$ and $x$ is near $x_{0}$. Namely, $\mathrm{d} \mathcal{A}_{\lambda_{0}}(x)[L v]=\mathrm{d} \mathcal{A}_{\lambda_{0}}(x)[L v]-\mathrm{d} \mathcal{A}_{\lambda_{0}}\left(x_{0}\right)[L v]=$ $\mathrm{d}^{2} \mathcal{A}_{\lambda_{0}}\left(x_{0}+\bar{t} v\right)[v, L v]=\left\langle S_{\lambda_{0}, x_{0}+\bar{t} v} L v, v\right\rangle_{1}$ for some $\bar{t} \in[0,1]$. Since $S_{\lambda_{0}, x_{0}} L$ is a positive isomorphism of $\mathcal{H}_{1}$, then by continuity $S_{\lambda_{0}, x_{0}+\bar{t} v} L$ is a positive isomorphism for $x$ near $x_{0}$. Thus, $\left\langle S_{\lambda_{0}, x_{0}+\bar{t} v} L v, v\right\rangle_{1}>0$ for $v \neq 0$, and (c) holds.

By Proposition 2.2, $\mathcal{A}_{\lambda_{0}}$ satisfies the Palais-Smale condition in a closed neighborhood of $x_{0}$ in $\mathfrak{M}$. Using the transversality of $\mathcal{S}$ to the critical orbit $\mathcal{O}$, it follows that also the restriction of $\mathcal{A}_{\lambda_{0}}$ to a closed neighborhood of $x_{0}$ in $\mathcal{S}$ satisfies the Palais-Smale condition. We can therefore apply Chang's result on Morse theory in Banach manifolds applied to the restriction of $\mathcal{A}_{\lambda_{0}}$ to $\mathcal{S}$; by [11, Theorem 1], we have:

$$
H_{k}\left(\mathcal{A}_{\lambda_{0}}^{c} \cap \mathcal{S},\left(\mathcal{A}_{\lambda_{0}}^{c} \cap \mathcal{S}\right) \backslash\left\{x_{0}\right\} ; \mathbb{Z}_{2}\right) \cong \begin{cases}\mathbb{Z}_{2}, & \text { if } k=\mu \\ 0, & \text { if } k \neq \mu\end{cases}
$$

With this, in order to compute the critical groups of the orbit $\mathcal{O}$ we can use an abstract result on the homology of fiber bundles. One has a bundle pair $(E, \dot{E})$ on the manifold $\mathcal{O}$, where $E=\mathcal{A}_{\lambda_{0}}^{c} \cap U$ has typical fiber $F=\mathcal{A}_{\lambda_{0}}^{c} \cap \Sigma_{x_{0}}$ and $\dot{E}=\left(\mathcal{A}_{\lambda_{0}}^{c} \cap U\right) \backslash \mathcal{O}$ has typical fiber $\dot{F}=\left(\mathcal{A}_{\lambda_{0}}^{c} \cap \Sigma_{x_{0}}\right) \backslash\left\{x_{0}\right\}$. Using the Leray-Hirsch theorem (see [42, Theorem 9]), we have the following isomorphism:

$$
H_{k}\left(E, \dot{E} ; \mathbb{Z}_{2}\right) \cong \bigoplus_{i=0}^{n} H_{i}\left(F, \dot{F} ; \mathbb{Z}_{2}\right) \otimes_{\mathbb{Z}_{2}} H_{n-i}\left(\mathcal{O} ; \mathbb{Z}_{2}\right) .
$$

Leray-Hirsch theorem uses two assumptions that are easily verified in our case. First, the relative homology $H_{i}\left(F, \dot{F} ; \mathbb{Z}_{2}\right)$ has to be finite dimensional for all $i$. Second, a technical condition called cohomology extension of the fiber has to be satisfied. When $E$ is (homotopic to) an open neighborhood of the zero section of a vector bundle and $\dot{E}$ is $E$ minus the zero section, then the cohomology extension of the fiber exists always when the coefficient field is $\mathbb{Z}_{2}$. From (2.3) and (2.4) we compute easily:

$$
\begin{aligned}
\mathfrak{H}_{k}\left(\mathcal{O} ; \mathbb{Z}_{2}\right) & =H_{k}\left(\mathcal{A}_{\lambda_{0}}^{c} \cap U,\left(\mathcal{A}_{\lambda_{0}}^{c} \cap U\right) \backslash \mathcal{O} ; \mathbb{Z}_{2}\right) \\
\cong & \bigoplus_{i=0}^{k} H_{i}\left(\mathcal{A}_{\lambda_{0}}^{c} \cap \Sigma_{x_{0}},\left(\mathcal{A}_{\lambda_{0}}^{c} \cap \Sigma_{x_{0}}\right) \backslash\left\{x_{0}\right\} ; \mathbb{Z}_{2}\right) \otimes_{\mathbb{Z}_{2}} H_{k-i}\left(\mathcal{O} ; \mathbb{Z}_{2}\right) \\
\cong & \bigoplus_{i=0}^{k} H_{i}\left(\mathcal{A}_{\lambda_{0}}^{c} \cap \mathcal{S},\left(\mathcal{A}_{\lambda_{0}}^{c} \cap \mathcal{S}\right) \backslash\left\{x_{0}\right\} ; \mathbb{Z}_{2}\right) \otimes_{\mathbb{Z}_{2}} H_{k-i}\left(\mathcal{O} ; \mathbb{Z}_{2}\right) \\
= & H_{\mu}\left(\mathcal{A}_{\lambda_{0}}^{c} \cap \mathcal{S},\left(\mathcal{A}_{\lambda_{0}}^{c} \cap \mathcal{S}\right) \backslash\left\{x_{0}\right\} ; \mathbb{Z}_{2}\right) \otimes_{\mathbb{Z}_{2}} H_{k-\mu}\left(\mathcal{O} ; \mathbb{Z}_{2}\right) \cong H_{k-\mu}\left(\mathcal{O} ; \mathbb{Z}_{2}\right) .
\end{aligned}
$$

This concludes the proof.

2.7. Equivariant constrained bifurcation. In the variational setup (A1)-(A5), let us now assume that we have a path of constrained critical orbits, as follows: 
(B1) $[a, b] \ni r \mapsto \lambda_{r} \in \mathbb{R}$ is a map of class $C^{1}$, with derivative $\lambda_{r}^{\prime}>0$ for all $r$;

(B2) $[a, b] \ni r \mapsto x_{r} \in \mathfrak{M}$ is a map of class $C^{1}$, and $x_{r}$ is a critical point of $\mathcal{A}_{r}=\mathcal{A}_{\lambda_{r}}$ for all $r$.

The assumption $\lambda_{r}^{\prime}>0$ in (B1) implies that the image of the map $\lambda$ is an interval $[c, d]$, and that there exists the inverse function $[c, d] \ni \lambda \mapsto r_{\lambda} \in[a, b]$.

We say that an instant $\bar{r} \in[a, b]$ is an constrained critical orbit bifurcation instant if there exists a sequence $r_{n}$ tending to $\bar{r}$, and a sequence $x_{n} \in \mathfrak{M}$ tending to $x_{\bar{r}}$ as $n$ tends to infinity, such that:

- $\mathrm{d} \mathcal{A}_{\lambda_{r_{n}}}\left(x_{n}\right)=0$;

- $x_{n} \notin \mathcal{O}\left(x_{r_{n}}, G\right)$,

for all $n \in \mathbb{N}$. In other words, $\bar{r}$ is a constrained critical orbit bifurcation instant if arbitrarily close to $\mathcal{O}\left(x_{\bar{r}}, G\right)$ one can find other constrained critical orbits that do not belong to the given path of constrained critical orbits.

Under suitable assumptions, degeneracy is a necessary condition for bifurcation. This is obtained by refining the result of Lemma 2.1, as follows:

Proposition 2.4. Consider the variational setup (A1)-(A5), and (B1), (B2). Let $\bar{r} \in] a, b[$ be fixed; assume that $\mathcal{O}\left(x_{\bar{r}}, G\right)$ is a nondegenerate critical orbit of $\mathcal{A}_{\lambda_{\bar{r}}}$ and that $(H F-A)$ holds around every point of $\mathcal{O}\left(x_{\bar{r}}, G\right)$. If:

(C1) the (connected component of the identity of the) isotropy group $G_{x_{r}}$ is constant for $r$ near $\bar{r}$;

(C2) the curve $r \mapsto x_{r}$ is not tangent to $\mathcal{O}\left(x_{\bar{r}}, G\right)$ at $x_{\bar{r}}$.

Then:

(a) for $\varepsilon>0$ small enough, the set

$$
\mathcal{O}_{\varepsilon}=\bigcup_{r \in] \bar{r}-\varepsilon, \bar{r}+\varepsilon[}\left\{\lambda_{r}\right\} \times \mathcal{O}\left(x_{r}, G\right)
$$

is a $C^{1}$-submanifold of $\mathbb{R} \times \mathfrak{M}$ of dimension $d+1$, where $d=\operatorname{dim}\left(G / G_{x_{\bar{r}}}\right)$;

(b) for $(\lambda, x)$ near $\left(\lambda_{\bar{r}}, \mathcal{O}\left(x_{\bar{r}}, G\right)\right), x$ is a critical point of $\mathcal{A}_{\lambda}$ if and only if $(\lambda, x) \in$ $\mathcal{O}_{\varepsilon}$.

In particular, constrained critical orbit bifurcation does not occur at $\bar{r}$.

Proof. By equivariance, it suffices to study the problem in the neighborhood $W$ of $\left(\lambda_{\bar{r}}, x_{\bar{r}}\right)$ where the thesis of Lemma 2.1 holds. The set $\mathcal{O}_{\varepsilon} \cap W$ is contained in the $(d+1)$ dimensional $C^{1}$-submanifold $\mathcal{D}$ consisting of pseudo-critical points, whose existence in proven in Lemma 2.1. Moreover, $\mathcal{O}_{\varepsilon} \cap W$ is foliated by the sets $\mathcal{O}\left(x_{r_{\lambda}}, G\right) \cap W$, for $\lambda \in] \lambda_{\bar{r}-\varepsilon}, \lambda_{\bar{r}+\varepsilon}$, that are submanifolds of dimension $d$ of $\mathbb{R} \times \mathfrak{M}$ by (A5) and (C1), and it contains the curve $\lambda \mapsto x_{r_{\lambda}}$ which not tangent to the orbit $\mathcal{O}\left(x_{\bar{r}}, G\right)$ by (C2). The foliation $\lambda \mapsto \mathcal{O}\left(x_{r_{\lambda}}, G\right)$ is continuous, by $(\mathrm{C} 1)$ and the fact the action of $G$ on $\mathfrak{M}$ is continuous. It follows that $\mathcal{O}_{\varepsilon} \cap W=\mathcal{D} \cap(] \lambda_{\bar{r}-\varepsilon}, \lambda_{\bar{r}+\varepsilon}[\times \mathfrak{M})$ for $\varepsilon>0$ small enough, and the conclusion follows immediately from Lemma 2.1 .

Remark 2.5. When $\mathfrak{M}$ is a Banach space and the action of $G$ is by linear isomorphisms of $\mathfrak{M}$, the result can be proven using a $G$-equivariant version of the infinite dimensional Implicit Function Theorem, as proved for instance in [16, 17, 35], replacing assumption $(\mathrm{C} 1)$ with an the algebraic assumption that the isotropic representation of $G_{x_{\bar{r}}}$ on $T_{x_{\bar{r}}} \mathcal{O}\left(x_{\bar{r}}, G\right)$ should not have non zero fixed point. This is also equivalent to the fact that the stabilizer $G_{x_{\bar{r}}}$ has the same dimension of its normalizer in $G$.

Proposition 2.6. Consider the variational setup (A1)-(A5), and (B1), (B2). Let I $\subset$ $[a, b]$ be an interval such that, for all $r \in I, \mathcal{O}\left(x_{r}, G\right)$ is nondegenerate, the critical orbit $\mathcal{O}\left(x_{r}, G\right)$ has constant dimension, and such that $(H F-B)$ holds around every $x_{r}$. Then, the strong Morse index $\mathfrak{i}_{\text {Morse }}^{\mathrm{s}}\left(\mathcal{O}\left(x_{r}, G\right)\right)$ is constant on $I$. 
Proof. The integer valued functions index and index plus nullity are respectively lower and upper semi-continuous in the space of essentially positive Fredholm operators on a Hilbert space (see for instance [24, Corollary 2.8]). Our assumptions imply that the nullity of the second variation of the functionals $\mathcal{A}_{\lambda_{r}}$ is constant on $I$. Thus, the index function $\mathfrak{i}_{\text {Morse }}^{\mathrm{s}}\left(\mathcal{O}\left(x_{r}, G\right)\right)$ is both lower and upper semi-continuous on $I$, hence constant.

Degeneracy is only a necessary condition for orbit bifurcation. In order to guarantee bifurcation a sufficient condition is that the degeneracy occurs at an instant $\bar{r}$ where the critical groups of the orbit $\mathcal{O}\left(x_{\bar{r}}, G\right)$ have a discontinuity. Under assumptions (C1) and (C2), such discontinuity occurs at the instants where the strong Morse index has a jump. There are several results available for equivariant bifurcation, but they don't quite fit into our framework; for instance, in [41] it is studied equivariant bifurcation from a branch of isolated critical points, i.e., from critical orbits consisting of just one point. In view to our application, in which some global regularity assumptions will be dropped, it will be convenient to give a proof using a non smooth approach.

Theorem 2.7. Consider the variational setup (A1)—(A5), and (B1), (B2); for $r \in[a, b]$ set:

$$
\begin{aligned}
& \qquad \mu_{r}=\mathfrak{i}_{\text {Morse }}^{\mathrm{s}}\left(\mathcal{O}\left(x_{r}, G\right)\right) \\
& \text { Let } \bar{r} \in] a, b[\text { be a given instant, and assume the following: } \\
& \text { - axiom }(H F-A) \text { holds at all points of } \mathcal{O}\left(x_{\bar{r}}, G\right) ; \\
& \text { - axiom }(H F-B) \text { holds in a neighborhood of } \mathcal{O}\left(x_{\bar{r}}, G\right) ; \\
& \text { - assumptions }(C 1) \text { and }(C 2) \text { hold; }
\end{aligned}
$$

(D1) for $r \neq \bar{r}$ the critical orbit $\mathcal{O}\left(x_{r}, G\right)$ is nondegenerate;

(D2) for $\varepsilon>0$ small, $\mu_{\bar{r}-\varepsilon} \neq \mu_{\bar{r}+\varepsilon}$.

Then, $\bar{r}$ is a constrained critical orbit bifurcation instant.

Proof. Note that, by Proposition 2.6, $\mu_{r}$ is constant on the left and on the right of $\bar{r}$, and thus assumption (D2) does not depend on the choice of $\varepsilon$ small enough. By Proposition 2.2, for $r$ near $\bar{r}$ the functional $\mathcal{A}_{\lambda_{r}}$ satisfies the Palais-Smale condition on a closed neighborhood of the orbit $\mathcal{O}\left(x_{\bar{r}}, G\right)$. Moreover, the assumption (D2) on the jump of the Morse index and the fact that, by $(\mathrm{C} 1)$, the critical orbits $\mathcal{O}_{r}=\mathcal{O}\left(x_{r}, G\right)$, have the same dimension $d$ (in fact, they are all diffeomorphic if the whole isotropy group $G_{x_{r}}$ is constant near $\bar{r})$, we have that the sequence of critical groups $\mathfrak{H}_{*}\left(\mathcal{O}_{r}, \mathbb{Z}_{2}\right)$ has a jump at $r=\bar{r}$. Namely, set $\mu_{ \pm}=\mu_{\bar{r} \pm \varepsilon}$, and assume $\mu_{+}>\mu_{-}$. Then, using Proposition 2.3, for $\varepsilon>0$ small enough we have:

$$
\mathfrak{H}_{d+\mu_{+}}\left(\mathcal{O}_{\bar{r}+\varepsilon}, \mathbb{Z}_{2}\right) \cong H_{d}\left(\mathcal{O}_{\bar{r}+\varepsilon}, \mathbb{Z}_{2}\right) \cong \mathbb{Z}_{2}
$$

while

$$
\mathfrak{H}_{d+\mu_{+}}\left(\mathcal{O}_{\bar{r}-\varepsilon}, \mathbb{Z}_{2}\right) \cong H_{d+\mu_{+}-\mu_{-}}\left(\mathcal{O}_{\bar{r}-\varepsilon}, \mathbb{Z}_{2}\right)=\{0\} .
$$

Similarly, if $\mu_{+}<\mu_{-}$, then $\mathfrak{H}_{d+\mu_{-}}\left(\mathcal{O}_{\bar{r}+\varepsilon}, \mathbb{Z}_{2}\right)=0$ while $\mathfrak{H}_{d+\mu_{-}}\left(\mathcal{O}_{\bar{r}-\varepsilon}, \mathbb{Z}_{2}\right) \cong \mathbb{Z}_{2}$.

Set $\bar{\lambda}=\lambda_{\bar{r}}$ and let $\varepsilon^{\prime}$ be such that $\left[\bar{\lambda}-\varepsilon^{\prime}, \bar{\lambda}+\varepsilon^{\prime}\right] \subset\left[\lambda_{\bar{r}-\varepsilon}, \lambda_{\bar{r}+\varepsilon}\right]$. An equivariant version $^{2}$ of the stability result for critical groups given in [14, Theorem 5.2] says that, when the local Palais-Smale condition holds in some closed neighborhood $W$ of $\mathcal{O}_{\bar{r}}$, if the following two assumptions are satisfied:

- the only critical orbit of $\mathcal{A}_{\lambda}$ in $W$ is $\mathcal{O}_{r_{\lambda}}$ for $\lambda \in\left[\bar{\lambda}-\varepsilon^{\prime}, \bar{\lambda}+\varepsilon^{\prime}\right]$;

- the critical orbits $\mathcal{O}_{\bar{r}-\varepsilon}$ and $\mathcal{O}_{\bar{r}+\varepsilon}$ are nondegenerate, then

$$
\mathfrak{H}_{*}\left(\mathcal{O}_{\bar{r}-\varepsilon}, \mathbb{Z}_{2}\right)=\mathfrak{H}_{*}\left(\mathcal{O}_{\bar{r}+\varepsilon}, \mathbb{Z}_{2}\right)
$$

\footnotetext{
${ }^{2}$ A complete proof of a stability result for the critical groups in the case of critical submanifolds, that can be adapted to our situation, is found in the unpublished work [18].
} 
Clearly, (2.7) is in contradiction with (2.5) and (2.6), which shows that, for every sufficiently small closed neighborhood of $\mathcal{O}_{\bar{r}}$ and every sufficiently small $\varepsilon^{\prime}>0$, some $\mathcal{A}_{\lambda}$, with $\lambda \in\left[\bar{\lambda}-\varepsilon^{\prime}, \bar{\lambda}+\varepsilon^{\prime}\right]$, admits a critical orbit contained in $W$ and distinct from $\mathcal{O}_{r_{\lambda}}$. This means that critical orbit bifurcation occurs at $r=\bar{r}$.

Observe that, by (2.2), assumption (D2) in Theorem 2.7 holds if the weak Morse index has a jump of at least two at $\bar{r}$, i.e., if:

$$
\left|\mathfrak{i}_{\text {Morse }}^{\mathrm{w}}\left(\mathcal{O}\left(x_{\bar{r}-\varepsilon}, G\right)\right)-\mathfrak{i}_{\text {Morse }}^{\mathrm{w}}\left(\mathcal{O}\left(x_{\bar{r}+\varepsilon}, G\right)\right)\right| \geq 2 .
$$

Theorem 2.7 will in fact be used in the present paper under the slightly weaker assumption that the manifold $\mathfrak{M}$ has only a local differentiable structure defined by an atlas of charts that are continuously compatible, and that the functions $\mathcal{A}$ and $\mathcal{V}$ are smooth in these local charts. The purely topological nature of the global aspects of the proof of Theorem 2.7, namely, the computation of the local Morse invariants (Proposition 2.3) and the stability result for critical groups, makes it clear that the result of Theorem 2.7 holds under these more general assumptions.

\section{ON THE VARIATIONAL PROBLEM OF CMC HYPERSURFACES}

Let us formalize the question of obtaining constant mean curvature hypersurfaces of a Riemannian manifold as critical points of the area functional restricted to volume preserving variations, in the spirit of [7]. Let $(N, g)$ be a connected Riemannian manifold, and let $M$ be a connected compact differentiable manifold with $\operatorname{dim}(N)=\operatorname{dim}(M)+1$. We will assume for simplicity that $M$ and $N$ are oriented, although the entire theory can be developed also in the non orientable case. Let $\operatorname{vol}_{g}$ denote the volume form on $N$ associated to the metric $g$.

3.1. The manifold of unparameterized embeddings. Let $k \geq 2$ and $\alpha \in] 0,1[$ be fixed. Consider the smooth Banach manifold $C^{k, \alpha}(M, N)$ of all maps $x: M \rightarrow N$ of Hölder class $C^{k, \alpha}$, and let $\operatorname{Emb}(M, N)$ be the open subset of $C^{k, \alpha}(M, N)$ consisting of embeddings. For the main results of the present paper, we will consider the case $k=2$. The set $\widetilde{\operatorname{Emb}}(M, N)$ is the set of equivalence classes $\{[x]: x \in \operatorname{Emb}(M, N)\}$, where two embeddings $x, y \in \operatorname{Emb}(M, N)$ are equivalent if there exists a diffeomorphism $\phi: M \rightarrow M$ such that $x=y \circ \phi$. Elements of $\widetilde{\operatorname{Emb}}(M, N)$ are called unparameterized embeddings of class $C^{k, \alpha}$ of $M$ into $N$. The topology of $\overparen{\operatorname{Emb}}(M, N)$ is the quotient topology induced from $\operatorname{Emb}(M, N)$. The geometrical structure of $\widetilde{\operatorname{Emb}}(M, N)$ is studied in [5]. Let us recall here that $\widetilde{\operatorname{Emb}}(M, N)$ has a natural atlas of charts that makes into an infinite dimensional topological Banach manifold. The charts of this atlas are of the following form. Given a smooth (i.e., $C^{\infty}$ ) embedding $x: M \rightarrow N$, there exists an open neighborhood $\widetilde{\mathcal{U}}_{x}$ of $[x]$ in $\widehat{\operatorname{Emb}}(M, N)$ and an open neighborhood $\mathcal{W}_{x}$ of the zero section of the Banach space of $C^{k, \alpha}$ section of the normal bundle $x^{\perp}$ of $x$, and a bijection $\widetilde{\Phi}_{x}: \widetilde{\mathcal{U}}_{x} \rightarrow \widetilde{\mathcal{W}}_{x}$ defined by $\widetilde{\Phi}_{x}([y])=u$, where the map $z: M \rightarrow N$ given by $z(p)=\exp _{x(p)}(u(p))$, $p \in M$, is an embedding of class $C^{k, \alpha}$ which is equivalent to $y$.

As $x$ runs in the set of smooth embeddings, the maps $\widetilde{\Phi}_{x}$ form an atlas of charts for $\widetilde{\operatorname{Emb}}(M, N)$ that are continuously compatible. If $F: \operatorname{Emb}(M, N) \rightarrow \mathbb{R}$ is a smooth function which is invariant by reparamaterization, i.e., $F(x \circ \phi)=F(x)$ for all diffeomorphism $\phi: M \rightarrow M$, then the induced map $\widetilde{F}: \widetilde{\operatorname{Emb}}(M, N) \rightarrow \mathbb{R}$ is smooth in every local chart, i.e., $\widetilde{F} \circ \widetilde{\Phi}_{x}^{-1}$ is smooth for all $x$. Thus, one has a well defined notion of critical point for a smooth function $\widetilde{F}$ on $\widetilde{\operatorname{Emb}}(M, N)$, as well as a natural notion of second derivative $\mathrm{d}^{2} \widetilde{F}\left(\left[x_{0}\right]\right)$ at a critical point $\left[x_{0}\right]$. 
Moreover, the smooth action of the isometry group of $(N, g)$ on $\operatorname{Emb}(M, N)$ by right composition induces a continuous action on $\widetilde{\operatorname{Emb}}(M, N)$. Given a smooth $x$, the $[x]$-orbit of this action is a smooth submanifold of $\widetilde{\operatorname{Emb}}(M, N)$ in local charts.

Details are found in [5].

3.2. The area functional. Given an embedding $x: M \rightarrow N$, one can define the area $\mathcal{A}(x)$ of $x$ as the volume of $M$ relatively to the volume form $x^{*}\left(\operatorname{vol}_{g}\right)$, which is the pullback of $\operatorname{vol}_{g}$ by $x$ :

$$
\mathcal{A}(x)=\int_{M} x^{*}\left(\operatorname{vol}_{g}\right)
$$

If an auxiliary Riemannian metric $h$ is fixed on $M$, then $\mathcal{A}(x)$ can be written more explicitly as:

$$
\mathcal{A}(x)=\int_{M}\left(\operatorname{det}\left(\mathrm{d} x(p)^{*} \mathrm{~d} x(p)\right)\right)^{1 / 2} \operatorname{vol}_{h},
$$

where $\mathrm{d} x(p)^{*}$ is the adjoint of the linear map $\mathrm{d} x(p): T_{p} M \rightarrow T_{x(p)} N$, adjoint taken relatively to the scalar products $h_{p}$ on $T_{p} M$ and $g_{x(p)}$ on $T_{x(p)} N$. Note that $\mathcal{A}$ can be seen as a functional on the manifold $\operatorname{Emb}(M, N)$, and it is invariant by the group of diffeomorphisms of $M$; thus, $\mathcal{A}$ gives a well defined functional on the quotient space $\widetilde{\operatorname{Emb}}(M, N)$, still denoted by $\mathcal{A}$ with a slight abuse of notations. Using the local charts of $\widetilde{\operatorname{Emb}}(M, N)$ described above, $\mathcal{A}$ is a smooth function in the neighborhood of every smooth embedding. More precisely:

Proposition 3.1. Let $x$ be a $C^{\infty}$ embedding of $M$ into $N$; let $\left(\widetilde{\mathcal{U}}_{x}, \widetilde{\Phi}_{x}\right)$ be the local chart of $\widetilde{\operatorname{Emb}}(M, N)$ around $[x]$ described in Subsection 3.1. Then, the map $\mathcal{A}_{x}=\mathcal{A} \circ \widetilde{\Phi}_{x}^{-1}$ : $\widetilde{\Phi}_{x}\left(\widetilde{\mathcal{U}}_{x}\right) \rightarrow \mathbb{R}^{+}$is smooth, and $u$ is a critical point of this functional if and only if $\widetilde{\Phi}_{x}^{-1}(u)$ is the class $[y] \in \widetilde{\mathcal{U}}_{x} \subset \widetilde{\operatorname{Emb}}(M, N)$ of a minimal embedding $y: M \rightarrow N$.

Proof. First, note that the functional $\mathcal{A}$ is smooth on $\operatorname{Emb}(M, N)$. Namely, in the local charts described in Subsection 3.1, $\mathcal{A}$ is given by the composition of a nonlinear first order differential operator defined on $C^{k, \alpha}$-sections of $x^{*}(T N)$ with the linear map

$$
C^{0}(M, \mathbb{R}) \ni f \longmapsto \int_{M} f \operatorname{vol}_{h} \in \mathbb{R} .
$$

Second, observe that $\mathcal{A}$ is invariant by diffeomorphisms of $M$, i.e., the area of an embedding does not depend on its parametrization. Smoothness of $\mathcal{A}_{x}$ follows now easily using the results of [5]. It is well known (see for instance [30]) that 0 is a critical point of $\mathcal{A}_{x}$ if and only if $x$ is a minimal embedding.

3.3. Volume of a region with boundary $\mathbf{x}(\mathbf{M})$. Let us now look at the variational problem of constant mean curvature embeddings. Before we go into the general case, let us first discuss an instructive problem of establishing when a given embedding $x: M \rightarrow N$ has image which is the boundary of an open subset of $N$. Equivalently, denoting by $M_{0} \subset N$ the image $x(M)$, we want to know when the open set $N \backslash M_{0}$ has two connected components. First, observe that a necessary condition for this is that $M_{0}$ is transversally oriented in $N$, i.e., the normal bundle $T M_{0}^{\perp}$ must be orientable. Thus, let us assume that $M_{0}$ is transversally oriented.

The number of connected components is the rank of the free abelian group $\widetilde{H}_{0}\left(N \backslash M_{0}\right)$ plus 1 , where $\widetilde{H}_{0}$ denotes the reduced singular homology group. The long exact reduced homology sequence of the pair $\left(N, N \backslash M_{0}\right)$ gives:

$$
H_{1}(N) \longrightarrow H_{1}\left(N, N \backslash M_{0}\right) \longrightarrow \tilde{H}_{0}\left(N \backslash M_{0}\right) \longrightarrow \tilde{H}_{0}(N)=0 .
$$

Since $M_{0}$ is closed in $N$, we can use excision and replace $N$ with a tubular neighborhood of $M_{0}$ in the term $H_{1}\left(N, N \backslash M_{0}\right)$; it follows that $H_{1}\left(N, N \backslash M_{0}\right)$ is the same as the relative 
homology $H_{1}\left(T M_{0}^{\perp}, T M_{0}^{\perp} \backslash \mathbf{0}\right)$, where $T M_{0}^{\perp}$ is the normal bundle of $M_{0}$ and $\mathbf{0}$ is its zero section. The calculation of homology of vector bundles is well known, see for instance [42]; since $M_{0}$ is transversally oriented, i.e., the vector bundle $T M_{0}^{\perp}$ is orientable, then $H_{1}\left(T M_{0}^{\perp}, T M_{0}^{\perp} \backslash \mathbf{0}\right)$ is isomorphic to $H_{0}\left(M_{0}\right) \cong \mathbb{Z}$. Thus, we have an exact sequence:

$$
H_{1}(N) \longrightarrow \mathbb{Z} \longrightarrow \widetilde{H}_{0}\left(N \backslash M_{0}\right) \longrightarrow 0 \text {. }
$$

A generator of the group $\mathbb{Z}$ above is a curve in $N$ that intercepts once and transversally $M_{0}$. Since $\widetilde{H}_{0}\left(N \backslash M_{0}\right)$ is free, the only options for the image of the map $H_{1}(N) \rightarrow \mathbb{Z}$ are that this image is either all $\mathbb{Z}$ or zero. When this image is $\mathbb{Z}$, then $N \backslash M_{0}$ is connected; when the image is zero, then $N \backslash M_{0}$ has two connected components. Thus, we have the following:

Lemma 3.2. Let $M$ and $N$ be compact connected manifolds, with $\operatorname{dim}(N)=\operatorname{dim}(M)+1$. Then, the set:

$$
\widetilde{\operatorname{Emb}}^{o}(M, N)=\{[x] \in \widetilde{\operatorname{Emb}}(M, N): N \backslash x(M) \text { has two connected components }\}
$$

is open in $\widetilde{\operatorname{Emb}}(M, N)$.

Proof. The openness of $\operatorname{Emb}^{o}(M, N)$ in $\operatorname{Emb}(M, N)$ follows readily from the discussion above, observing that the orientability of the normal bundle of an embedding $x$ : $M \rightarrow N$ is stable by $C^{1}$-perturbations, while the homology class of $x$ is stable by $C^{0}$ perturbations. Then, also $\widetilde{\operatorname{Emb}}^{o}(M, N)$ is open in $\widetilde{\operatorname{Emb}}(M, N)$, because $\tilde{\pi}$ is a quotient map and $\widetilde{\pi}^{-1}\left(\widetilde{\operatorname{Emb}}_{1}^{o}(M, N)\right)=\operatorname{Emb}_{1}^{o}(M, N)$.

If $x: M \rightarrow N$ is a transversally oriented $C^{1}$-embedding, thus $[x] \in \widetilde{\operatorname{Emb}^{o}}(M, N)$, then one can write $N \backslash x(M)=\Omega_{x}^{1} \bigcup \Omega_{x}^{2}$ as the disjoint union of two non empty connected open subsets of $N$. Using an orientation of the normal bundle $x^{\perp}$ that depends continuously by $C^{1}$-perturbations of $x$, one can define a continuous functions $\mathcal{V}^{1}$ and $\mathcal{V}^{2}$ in the connected component of $x$ in $\widetilde{\operatorname{Emb}}_{1}^{o}(M, N)$, by setting:

$$
\mathcal{V}^{i}(x)=\operatorname{volume}\left(\Omega_{x}^{i}\right)=\int_{\Omega_{x}^{i}} \operatorname{vol}_{g}, \quad i=1,2 .
$$

Clearly, $\mathcal{V}^{2}(x)=\operatorname{vol}(N)-\mathcal{V}^{1}(x)$.

3.4. A generalized volume functional. Now, we observe that can write an alternative expression for the volume function without an explicit reference to the set $\Omega_{x}^{1}$. Let us now assume that $M$ and $N$ are oriented; then, one has a canonical choice of a transverse orientation of $x(M)$. Let $U$ be an open subset $N$ that contains $x(M)$, and such that ${ }^{3}$ $U \neq N$; for instance, $U$ can be taken to be a small tubular neighborhood of $x(M)$. Then, the volume form $\operatorname{vol}_{g}$, which is closed, must be exact when restricted to $U$, because non compact manifolds have vanishing de Rham cohomology in the highest dimension. This means that there exists an $(n-1)$-form $\eta_{U}$ on $U$ such that $\mathrm{d} \eta_{U}=\operatorname{vol}_{g}$ on $U$. Then, one can define:

$$
\mathcal{V}(x)=\int_{M} x^{*}\left(\eta_{U}\right)
$$

such a function is immediately seen to be independent of the choice of the primitive $\eta_{U}$. By Stokes theorem, if $x(M)$ is the boundary of an open subset $\Omega$ of $N$, then $\mathcal{V}(x)$ coincides with the volume of $\Omega$ or of $N \backslash \Omega$ (depending on the orientation of $M$ ), and thus the function $\mathcal{V}$ is a natural extension of the functions $\mathcal{V}^{1}$ and $\mathcal{V}^{2}$ when no assumption is made on the number of connected components of $N \backslash x(M)$. Note however that, when $N \backslash x(M)$ has only one connected component, the value of the functional $\mathcal{V}$ does indeed depend on

\footnotetext{
${ }^{3}$ Note that, by Sard theorem, since $\operatorname{dim}(M)<\operatorname{dim}(N)$ and $x$ is of class $C^{1}$, then $x(M) \neq N$. Thus, one can take $U=N \backslash\{p\}$, where $p$ is any point of $N$ that does not belong to $x(M)$.
} 
the choice of the open subset $U$ of $N$ that contains $x(M)$. It can be observed also that $\mathcal{V}$ is invariant by right-composition with diffeomorphisms of $M$, so that it gives a well defined function on a neighborhood of $[x]$ in $\widetilde{\operatorname{Emb}}(M, N)$. We need a reformulation of some standard results of CMC embeddings (see [7]) in our abstract variational context:

Proposition 3.3. Let $x: M \rightarrow N$ be a transversally oriented smooth embedding, and let $U$ and $\eta$ be as above; consider the local chart $\widetilde{\Phi}_{x}$ of $\widetilde{\operatorname{Emb}}(M, N)$ having domain $\widetilde{\mathcal{U}}_{x}$. We will assume here that $k \geq 2$.

(a) The (locally defined) function $\mathcal{V}_{x}=\mathcal{V} \circ \widetilde{\Phi}_{x}^{-1}$ on $\widetilde{\Phi}_{x}\left(\widetilde{\mathcal{U}}_{x}\right)$ is smooth, and it has no critical points.

(b) The critical points of the function $\mathcal{A}_{x}=\mathcal{A} \circ \widetilde{\Phi}_{x}^{-1}$ subject to the constraint $\mathcal{V}_{x}=$ const. are smooth sections $u$ of $x^{\perp}$ such that $\widetilde{\Phi}_{x}(u)$ is the class $[y]$ of a smooth embedding $y: M \rightarrow N$ having constant mean curvature.

(c) If $[x]$ is a critical point of $\mathcal{A}_{x}=\mathcal{A} \circ \widetilde{\Phi}_{x}^{-1}$ subject to the constraint $\mathcal{V}_{x}=$ const., then its Lagrange multiplier $\lambda_{x}$ is equal to $m \cdot H_{x}$, where $H_{x}$ is the (constant) mean curvature of $x$.

(d) If $[x]$ is a critical point of $\mathcal{A}_{x}$ subject to the constraint $\mathcal{V}_{x}=$ const., then identifying ${ }^{4} \boldsymbol{\Gamma}\left(x^{\perp}\right)$ with $C^{k, \alpha}(M, \mathbb{R})$, the space of $C^{k, \alpha}$-functions on $M$, the second variation $\mathrm{d}^{2} \mathcal{A}_{x}(x)$ at the point $x$ is the symmetric bilinear form on $C^{k, \alpha}(M, \mathbb{R})$ corresponding to the quadratic form:

$$
Q_{x}(f)=\int_{M}-f \Delta f-\left(m \operatorname{Ric}_{N}\left(\vec{n}_{x}\right)+\left\|S_{x}\right\|^{2}\right) f^{2} \operatorname{vol}_{x},
$$

where $\Delta$ is the Laplacian on functions on $M$ relative to the induced metric $x^{*}(g)$, $\operatorname{Ric}_{N}\left(\vec{n}_{x}\right)$ is the Ricci curvature of $N$ in the direction $\vec{n}_{x}$, which is the positively oriented unit normal field of $x, S_{x}$ is the second fundamental form of $x$ in the direction $\vec{n}_{x},\|\cdot\|$ is the Hilbert-Schmidt norm of an operator defined by $g$, and $\mathrm{vol}_{x}$ is the volume form of the metric $x^{*}(g)$.

Proof. The smoothness of $\mathcal{V}$ follows by the same argument used in the proof of the smoothness of $\mathcal{A}$ in Proposition 3.1: $\mathcal{V}$ is smooth map on $\operatorname{Emb}(M, N)$ invariant by diffeomorphisms of $M$, as it is in local charts the composition of a nonlinear first order differential operator in the space of $C^{k, \alpha}$-sections of $x^{*}(T N)$ and the linear map given by integration on $M$. All the remaining statements are obtained directly from [7], observing that the functions $\mathcal{A}_{x}$ and $\mathcal{V}_{x}$ correspond to the functions $A$ and $V$ in [7]. ${ }^{5}$ The differential of $\mathcal{V}_{x}$ at $x$ is identified with the linear map $C^{k, \alpha}(M, \mathbb{R}) \ni f \mapsto \int_{M} f \operatorname{vol}_{x}$, which is not identically zero; thus, $x$ is not a critical point of $\mathcal{V}_{x}$, and it follows that $\mathcal{V}_{x}$ has no critical points, proving (a). Parts (b) and (c) follow by similar arguments from [7, Proposition 2.3] or [6, Proposition 2.7]. Part (d) is [7, Proposition 2.5].

Let us now look at the Fredholmness issues for the CMC variational problem; in order to comply with axiom (HF-B), we will henceforth assume that $k=2$, i.e., we will consider the manifold of unparameterized embeddings of class $C^{2, \alpha}$, with $\left.\alpha \in\right] 0,1[$. Given a

\footnotetext{
${ }^{4}$ Let $\vec{n}_{x}$ be positively orient unit normal field of the embedding $x$. An identification of $\boldsymbol{\Gamma}\left(x^{\perp}\right)$ and $C^{k, \alpha}(M, \mathbb{R})$ is given by $C^{k, \alpha}(M, \mathbb{R}) \ni f \mapsto f \cdot \vec{n}_{x} \in \boldsymbol{\Gamma}\left(x^{\perp}\right)$.

${ }^{5}$ In the language of [7], the volume function denoted by $V$ is associated to variations of $x$, i.e., to smooth maps $X:]-\varepsilon, \varepsilon[\times M \rightarrow N$, such that for all $t \in]-\varepsilon, \varepsilon\left[\right.$, the map $X_{t}:=X(t, \cdot): M \rightarrow N$ is an immersion, and such that $X_{0}=x$. Note that, since $x$ is an embedding, for $t$ near 0 also $X_{t}$ is an embedding. Associated to such a variation of $x$, it is defined in [7] the function $V(t)=\int_{[0, t] \times M} X^{*}\left(\operatorname{vol}_{g}\right)$, for $\left.t \in\right]-\varepsilon, \varepsilon[$. Now, using Stoke's Theorem:$$
V(t)=\int_{[0, t] \times M} X^{*}(\mathrm{~d} \eta)=\int_{[0, t] \times M} \mathrm{~d} X^{*}(\eta)=\int_{M}\left(X_{t}\right)^{*}(\eta)-\int_{M}\left(X_{0}\right)^{*}(\eta)=\mathcal{V}\left(X_{t}\right)-\mathcal{V}\left(X_{0}\right)
$$ 
constant mean curvature embedding $x: M \rightarrow N$, let $J: C^{2, \alpha}\left(x^{\perp}\right) \rightarrow C^{0, \alpha}\left(x^{\perp}\right)$ be the differential operator:

$$
J f=-\Delta f-\left(m \operatorname{Ric}_{N}\left(\vec{n}_{x}\right)+\left\|S_{x}\right\|^{2}\right) f .
$$

From part (d) of Proposition 3.3, the second variation of the functional $\mathcal{A}_{x}+\lambda_{x} \mathcal{V}_{x}$ is the symmetric bilinear form $B_{x}$ on $C^{2, \alpha}\left(x^{\perp}\right)$ given by:

$$
B_{x}\left(f_{1}, f_{2}\right)=\int_{M}\left(J f_{1}\right) \cdot f_{2} \operatorname{vol}_{x} .
$$

Proposition 3.4. The following statements hold:

(a) the functional $\mathcal{A}_{x}+\lambda_{x} \mathcal{V}_{x}$ satisfies the assumption (HF-A) in a neighborhood of $[x]$ in $\widetilde{\operatorname{Emb}}(M, N)$;

(b) the functional $\mathcal{A}_{x}+\lambda_{x} \mathcal{V}_{x}$ satisfies the assumption (HF-B) in a neighborhood of $[x]$ in $\widetilde{\operatorname{Emb}}(M, N)$.

Proof. Part (a) is well known, see for instance [40, Section 2], [46, §1.4], [47, Theorem 1.2 and $\S 7]$. The gradient map $H$ is defined in an open set of the Banach space $X=C^{2, \alpha}(M, \mathbb{R})$ and takes values in the Banach space $Y=C^{0, \alpha}(M, \mathbb{R})$. The Hilbert space $H$ is the Lebesgue space of square integrable functions on $M$ relatively to the metric defined by vol. An explicit formula for the map $H$ is irrelevant here; it is given by a quasi-linear second order elliptic differential operator, see $[46,47]$ for details.

For part (b), consider the embedding $C^{2, \alpha}(M, \mathbb{R}) \hookrightarrow H^{1}(M, \mathbb{R})$, where $H^{1}$ denotes the Sobolev space given by the Hilbert space completion of $C^{2, \alpha}(M, \mathbb{R})$ with respect to the pre-Hilbert space inner product

$$
\left\langle f_{1}, f_{2}\right\rangle_{H^{1}}=\int_{M}\left[f_{1} f_{2}+\nabla f_{1} \cdot \nabla f_{2}\right] \operatorname{vol}_{x}
$$

(gradients and inner products are relative to the Riemannian metric $x^{*}(g)$ on $M$ ). The symmetric bilinear form $B_{x}=\mathrm{d}^{2}\left[\mathcal{A}_{x}+\lambda_{x} \mathcal{V}_{x}\right](x)$ has a bounded extension to a symmetric bilinear form on $H^{1}(M, \mathbb{R})$, which is represented by an essentially positive self-adjoint operator on $H^{1}(M, \mathbb{R})$. Namely, partial integration in (3.4) gives;

$$
\begin{aligned}
B_{x}\left(f_{1}, f_{2}\right)=\int_{m} \nabla & f_{1} \cdot \nabla f_{2}-\left(m \operatorname{Ric}_{N}\left(\vec{n}_{x}\right)+\left\|S_{x}\right\|^{2}\right) f_{1} f_{2} \operatorname{vol}_{x} \\
& =\left\langle f_{1}, f_{2}\right\rangle_{H^{1}}-\int_{M}\left(m \operatorname{Ric}_{N}\left(\vec{n}_{x}\right)+\left\|S_{x}\right\|^{2}+1\right) f_{1} f_{2} \operatorname{vol}_{x},
\end{aligned}
$$

from which it follows that $B_{x}$ has a bounded extension to $H^{1}$. Now, such extension is represented by the operator $\mathrm{I}+K$, where I is the identity, and:

$$
\left\langle K f_{1}, f_{2}\right\rangle_{H^{1}}=-\int_{M}\left(m \operatorname{Ric}_{N}\left(\vec{n}_{x}\right)+\left\|S_{x}\right\|^{2}+1\right) f_{1} f_{2} \operatorname{vol}_{x} .
$$

The bilinear form on the right-hand side of this equality is continuous with respect to the $L^{2}$-topology, and this implies that $K$ is a compact operator, because the inclusion $H^{1} \hookrightarrow L^{2}$ is compact. Hence, $S=\mathrm{I}+K$ is essentially positive.

A function $f \in H^{1}(M, \mathbb{R})$ is an eigenfunction of $S$ if and only if it is a weak solution of the linear elliptic equation $J f=\mu f$ for some $\mu \in \mathbb{R}$. By standard elliptic regularity (see for instance [21, Chapter 8]), every such $f$ is of class $C^{\infty}$, and thus all the eigenspaces of $S$ are contained in $T_{[x]} \widetilde{\operatorname{Emb}}(M, N)$. This concludes the proof.

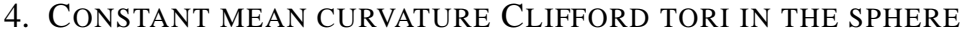

We will now finalize the proof of our main theorem, by applying the abstract results discussed in the first part of the paper. Let $0<j<m$ be fixed integers, and let us look at the case of CMC embeddings of the product of sphere $M=\mathbb{S}^{j} \times \mathbb{S}^{m-j}$ into the sphere $N=\mathbb{S}^{m+1}$ endowed with the round metric $g$. 
The Banach manifold $\mathfrak{M}$ is (an open subset of) $\widetilde{\operatorname{Emb}}(M, N)$, here $\alpha$ is any real number in ] 0,1 [. Consider the group $G=\mathrm{SO}(m+2)$, which is the connected component of the identity of $\mathrm{O}(m+2)=\operatorname{Iso}(N, g)$, acting by left-composition on $\mathfrak{M}$. For a given $x \in \widetilde{\operatorname{Emb}}(M, N)$, the stabilizer $G_{x}$ is given by the set of isometries $\psi \in \mathrm{SO}(m+1)$ that preserve the subset $x(M)$, i.e., such that $\psi(x(M))=x(M)$.

Consider the path $] 0,1\left[\ni r \mapsto\left[x_{r}^{m, j}\right] \in \widetilde{\operatorname{Emb}}(M, N)\right.$ of CMC Clifford tori defined in (1.1). If $g$ denotes the round metric of radius 1 on the sphere $\mathbb{S}^{m+1}$, the pull-back $\left(x_{r}^{m, j}\right)^{*}(g)$ is the product metric on $\mathbb{S}^{j} \times \mathbb{S}^{m-j}$ which is the round metric of radius $r$ on the factor $\mathbb{S}^{j}$ and the round metric of radius $\sqrt{1-r^{2}}$ on the factor $\mathbb{S}^{m-j}$. We will denote this Riemannian manifold by $\mathbb{S}^{j}(r) \times \mathbb{S}^{m-j}\left(\sqrt{1-r^{2}}\right)$.

Proposition 4.1. The connected component of the identity of the subgroup of $\mathrm{SO}(m+2)$ that stabilizes $\left[x_{r}^{m, j}\right]$ in $\widetilde{\operatorname{Emb}}(M, N)$ is $\mathrm{SO}(j+1) \times \mathrm{SO}(m-j+1)$ (embedded diagonally in $\mathrm{SO}(m+2))$.

Proof. Such a connected component is compact, and it obviously contains the product $\mathrm{SO}(j+1) \times \mathrm{SO}(m-j+1)$. On the other hand, it is not equal to $\mathrm{SO}(m+2)$ if $j$ and $m-j$ are positive. But $H=\mathrm{SO}(j+1) \times \mathrm{SO}(m-j+1)$ is a maximal connected subgroup of $\mathrm{SO}(m+2)$ (see for instance [19]), and thus it must be equal to the connected component of the identity of the stabilizer of $\left[x_{r}^{m, j}\right]$.

Corollary 4.2. The $\mathrm{SO}(m+2)$-orbit of the class $\left[x_{r}^{m, j}\right]$ in $\widetilde{\operatorname{Emb}}\left(\mathbb{S}^{j} \times \mathbb{S}^{m-j}, \mathbb{S}^{m}\right)$ is diffeomorphic to the Grassmannian of all $(j+1)$-dimensional oriented subspaces of $\mathbb{R}^{m+2}$, whose dimension is equal to $m+1+j(m-j)$.

Proof. The orbit of $\left[x_{r}^{m, j}\right]$ is diffeomorphic to the quotient

$$
\mathrm{SO}(m+2) /[\mathrm{SO}(j+1) \times \mathrm{SO}(m-j+1)],
$$

whose dimension is $\frac{1}{2}(m+2)(m+1)-\frac{1}{2} j(j+1)-\frac{1}{2}(m-j)(m-j+1)=m+1+$ $j(m-j)$.

The path of CMC Clifford tori is never tangent to the orbits:

Proposition 4.3. The curve $r \mapsto\left[x_{r}^{m, j}\right]$ is not tangent to the orbit $\mathrm{SO}(m+2)\left[x_{r_{0}}^{m, j}\right]$ for any value of $r_{0}$.

Proof. Fix $r_{0}$ and denote by $P^{\perp}: \boldsymbol{\Gamma}\left(\left(x_{r_{0}}^{m, j}\right)^{*}\left(T \mathbb{S}^{m+1}\right)\right) \rightarrow \boldsymbol{\Gamma}\left(\left(x_{r_{0}}^{m, j}\right)^{\perp}\right)$ the linear map that carries a vector field $V$ along $x_{r_{0}}^{m, j}$ to the normal field $V^{\perp}$ obtained by pointwise orthogonal projection onto the normal space of $x_{r_{0}}^{m, j}$. The tangent space to the orbit of $\left[x_{r}^{m, j}\right]$ is identified (via the local chart $\widetilde{\Phi}_{x_{r_{0}}^{m, j}}$ ) with the space:

$$
\left\{P^{\perp}\left(\mathfrak{h} \circ x_{r_{0}}^{m, j}\right): \mathfrak{h} \in \mathfrak{s o}(m+2)\right\},
$$

where $\mathfrak{s o}(m+2)$ is the Lie algebra of $\mathrm{SO}(m+2)$ (consisting of anti-symmetric matrices), while the tangent vector to the curve of CMC Clifford tori is:

where clearly:

$$
P^{\perp}\left(\left.\frac{\mathrm{d}}{\mathrm{d} r}\right|_{r=r_{0}} x_{r}^{m, j}\right),
$$

$$
\left.\frac{\mathrm{d}}{\mathrm{d} r}\right|_{r=r_{0}} x_{r}^{m, j}(p, q)=\left(p,-\frac{r_{0}}{\sqrt{1-r_{0}^{2}}} q\right), \quad \forall p \in \mathbb{S}^{j}, q \in \mathbb{S}^{m-j} .
$$

The thesis is equivalent to proving that there exists no $\mathfrak{h} \in \mathfrak{s o}(m+2)$ such that the vector field:

$$
\mathfrak{h} \circ x_{r_{0}}^{m, j}-\left.\frac{\mathrm{d}}{\mathrm{d} r}\right|_{r=r_{0}} x_{r}^{m, j}
$$


is everywhere tangent to the Clifford torus $x_{r_{0}}^{m, j}$. Writing:

$$
\mathfrak{h}=\left(\begin{array}{cc}
A & B \\
-B^{t} & C
\end{array}\right)
$$

where $A$ is an anti-symmetric $(j+1) \times(j+1)$ matrix, $B$ is a $(j+1) \times(m-j+1)$ matrix and $C$ is an anti-symmetric $(m-j+1) \times(m-j+1)$ matrix, then (4.1) is everywhere tangent to the Clifford torus if and only if:

$$
B q \cdot p=\frac{1}{\sqrt{1-r_{0}^{2}}}, \quad \forall p \in \mathbb{S}^{j}, q \in \mathbb{S}^{m-j} ;
$$

clearly, such condition is not satisfied by any $B$, which proves the thesis.

Let us now study the nondegeneracy and the Morse index of the CMC Clifford tori. Consider the sequences $\left(\beta_{i}(j)\right)_{i \geq 3}$ and $\left(\gamma_{l}(j, m)\right)_{l \geq 3}$ defined by:

$$
\beta_{i}(j)=(i-2)(j+i-1), \quad \gamma_{l}(j, m)=(l-2)(m-j+l-1) .
$$

It is easy to see that they are strictly increasing, and that:

$$
\lim _{i \rightarrow \infty} \beta_{i}(j)=\lim _{l \rightarrow \infty} \gamma_{l}(j, m)=+\infty .
$$

Define two sequences $\left(r_{i}^{m, j}\right)_{i \geq 3}$ and $\left(s_{l}^{m, j}\right)_{l \geq 3}$ by:

$$
r_{i}^{m, j}=\sqrt{\frac{\beta_{i}(j)}{m-j+\beta_{i}(j)}}, \quad s_{l}^{m, j}=\sqrt{\frac{j}{j+\gamma_{l}(j, m)}} ;
$$

the sequence $r_{i}^{m, j}$ is contained in $\left[\sqrt{\frac{j+2}{m+2}}, 1\left[\right.\right.$, it is strictly increasing, and $\lim _{i \rightarrow \infty} r_{i}^{m, j}=1$, while $s_{l}^{m, j}$ is contained in $\left.] 0, \sqrt{\frac{j}{m+2}}\right]$, it is strictly decreasing, and $\lim _{l \rightarrow \infty} s_{l}^{m, j}=0$.

Proposition 4.4. The following statements hold:

(a) the strong Morse index of $\left[x_{r}^{m, j}\right]$ is equal to the weak Morse index plus 1 ;

(b) the $\mathrm{SO}(m+1)$-critical orbits of $\left[x_{r}^{m, j}\right]$ is degenerate if and only if either $r=r_{i}^{m, j}$ for some $i \geq 3$ or $r=s_{l}^{m, j}$ for some $l \geq 3$;

(c) each degeneracy instant of the path of critical points $] 0,1\left[\ni r \mapsto\left[x_{r}^{m, j}\right]\right.$ determines a jump of the Morse index.

More precisely, at every degeneracy instant $r_{i}^{m, j}$, the jump of the Morse index is in absolute value equal to:

$$
\left(\begin{array}{c}
j+i-1 \\
i-1
\end{array}\right)-\left(\begin{array}{c}
j+1-3 \\
i-3
\end{array}\right)
$$

while at every degeneracy instant $s_{l}^{m, j}$, the jump of the Morse index is in absolute value equal to:

$$
\left(\begin{array}{c}
m-j+l-1 \\
l-1
\end{array}\right)-\left(\begin{array}{c}
m-j+l-3 \\
l-3
\end{array}\right) .
$$

Proof. The proof is based on a direct analysis of the spectrum of the Jacobi operator $J$ in (3.3), following [3]. The Ricci curvature of the sphere $\mathbb{S}^{m+1}$ is constant equal to 1; also the norm of the second fundamental form of the CMC Clifford embedding $x_{r}^{m, j}$ is constant, and the following formula holds:

$$
m \operatorname{Ric}_{N}\left(\vec{n}_{x_{r}^{m, j}}\right)+\left\|S_{x_{r}^{m, j}}\right\|^{2} \equiv \frac{j}{r^{2}}+\frac{m-j}{1-r^{2}} .
$$

Thus, the Jacobi operator takes the form:

$$
J=-\Delta_{r}^{j, m-j}-\left(\frac{j}{r^{2}}+\frac{m-j}{1-r^{2}}\right),
$$


where $\Delta_{r}^{j, m-j}$ is the Laplacian of the Riemannian manifold $\mathbb{S}^{j}(r) \times \mathbb{S}^{m-j}\left(\sqrt{1-r^{2}}\right)$. Thus, the spectrum of $J$ is given by the spectrum of $-\Delta^{j, m-j}$ shifted by $-\left(\frac{j}{r^{2}}+\frac{m-j}{1-r^{2}}\right)$; moreover, $J$ and $\Delta^{j, m-j}$ have the same eigenfunctions. Degeneracy and strong Morse index of the critical point $\left[x_{r}^{m, j}\right]$ is studied by counting the number of zero and negative eigenvalues of $J$ in the space of (smooth) real functions on $\mathbb{S}^{j} \times \mathbb{S}^{m-j}$. The weak Morse index is given by the index of the restriction of the quadratic form $\langle J f, f\rangle_{L^{2}}$ to the space of functions $f$ on $\mathbb{S}^{j} \times \mathbb{S}^{m-j}$ having vanishing integral.

An eigenvalue of $J$ has the form:

$$
\sigma_{i}+\rho_{l}-\left(\frac{j}{r^{2}}+\frac{m-j}{1-r^{2}}\right),
$$

where $\sigma_{i}$ is an eigenvalue of the Laplacian $\Delta_{r}^{j}$ of the sphere $\mathbb{S}^{j}(r)$ and $\rho_{l}$ is an eigenvalue of the Laplacian $\Delta_{\sqrt{1-r^{2}}}^{m-j}$ of the sphere $\mathbb{S}^{m-j}\left(\sqrt{1-r^{2}}\right)$. Moreover, if $M_{\sigma}$ and $M_{\rho}$ are the multiplicity of the eigenvalues $\sigma$ and $\rho$ respectively of $\Delta_{r}^{j}$ and of $\Delta_{\sqrt{1-r^{2}}}^{m-j}$, then the multiplicity of (4.4) is given by $\sum M_{\sigma} M_{\rho}$, where the sum is taken over all eigenvalues $\sigma$ and $\rho$ such that $\sigma+\rho=\sigma_{i}+\rho_{l}$.

The $\sigma_{i}$ 's and the $\rho_{l}$ 's form two strictly increasing unbounded sequences, and they have multiplicities denoted respectively by $M_{\sigma_{i}}$ and $M_{\rho_{l}}$ given by the following formulas:

$$
\begin{gathered}
\sigma_{i}=\frac{(i-1)(j+i-2)}{r^{2}}, \quad \rho_{l}=\frac{(l-1)(m-j+l-2)}{1-r^{2}} \\
M_{\sigma_{1}}=1, \quad M_{\sigma_{2}}=j+1, \quad M_{\sigma_{i}}=\left(\begin{array}{c}
j+i-1 \\
i-1
\end{array}\right)-\left(\begin{array}{c}
j+i-3 \\
i-3
\end{array}\right) \text { for } i \geq 3, \\
M_{\rho_{1}}=1, \quad M_{\rho_{2}}=m-j+1, \quad M_{\rho_{l}}=\left(\begin{array}{c}
m-j+l-1 \\
l-1
\end{array}\right)-\left(\begin{array}{c}
m-j+l-3 \\
l-3
\end{array}\right) \\
\text { for } l \geq 3 .
\end{gathered}
$$

The negative eigenvalue $-\left(\frac{j}{r^{2}}+\frac{m-j}{1-r^{2}}\right)=\sigma_{1}+\rho_{1}-\left(\frac{j}{r^{2}}+\frac{m-j}{1-r^{2}}\right)$ has multiplicity 1 , and its eigenspace consists of constant functions. This gives a contribution of 1 to the strong Morse index of $\left[x_{r}^{m, j}\right]$, but not to the weak Morse index. On the other hand, all the other eigenspaces of $J$ are $L^{2}$-orthogonal to the first eigenspace constisting of constant functions on $\mathbb{S}^{j} \times \mathbb{S}^{m-j}$, i.e., non constant eigenfunctions of $J$ have vanishing integral. This implies that the strong Morse index $\left[x_{r}^{m, j}\right]$ is equal to the weak Morse index plus 1, proving (a).

As to the degeneracy, let us observe that 0 is always an eigenvalue of $J$, because:

$$
\sigma_{2}+\rho_{2}-\left(\frac{j}{r^{2}}+\frac{m-j}{1-r^{2}}\right)=0,
$$

for all $r \in] 0,1\left[\right.$. Assuming that there is no other pair $(i, l) \neq(2,2)$ such that $\sigma_{i}+\rho_{l}-$ $\left(\frac{j}{r^{2}}+\frac{m-j}{1-r^{2}}\right)=0$, then the multiplicity of zero as an eigenvalue of $J$ is given by:

$$
M_{\sigma_{2}} M_{\rho_{2}}=(j+1)(m-j+1)=m+1+j(m-j) .
$$

By Corollary 4.2, this multiplicity equals the dimension of the critical orbit of $\left[x_{r}^{m, j}\right]$. Thus, nondegeneracy occurs exactly when there is no other 0 in the spectrum of $J$. Since the sequences $\sigma_{i}$ and $\rho_{l}$ are strictly increasing, it follows that other 0's in the spectrum of $J$ must be of the form

$$
\sigma_{1}+\rho_{l}-\left(\frac{j}{r^{2}}+\frac{m-j}{1-r^{2}}\right)
$$

for some $l \geq 3$, or of the form

$$
\sigma_{i}+\rho_{1}-\left(\frac{j}{r^{2}}+\frac{m-j}{1-r^{2}}\right)
$$


for some $i \geq 3$. Recalling (4.2) and (4.5), we compute explicitly:

$$
\begin{array}{r}
\sigma_{1}+\rho_{l}-\left(\frac{j}{r^{2}}+\frac{m-j}{1-r^{2}}\right)=\frac{(l-1)(m-j+l-2)}{1-r^{2}}-\left(\frac{j}{r^{2}}+\frac{m-j}{1-r^{2}}\right) \\
=\frac{r^{2}\left[j+\gamma_{l}(j, m)\right]-j}{r^{2}\left(1-r^{2}\right)}, \\
\sigma_{i}+\rho_{1}-\left(\frac{j}{r^{2}}+\frac{m-j}{1-r^{2}}\right)=\frac{(i-1)(j+i-2)}{r^{2}}-\left(\frac{j}{r^{2}}+\frac{m-j}{1-r^{2}}\right) \\
=\frac{\beta_{i}(j)-r^{2}\left[m-j+\beta_{i}(j)\right]}{r^{2}\left(1-r^{2}\right)} .
\end{array}
$$

The first expression above vanishes for $r \in] 0,1\left[\right.$ exactly when $r=s_{l}^{m, j}$, while the second expression vanishes for $r \in] 0,1$ [ exactly when $r=r_{i}^{m, j}$, see (4.3). This proves part (b).

For the proof of part (c), we will show that the jump of the Morse index at the instants $s_{l}^{m, j}$ is equal to the multiplicity $M_{\rho_{l}}$, while the jump of the Morse index at the instants $r_{i}^{m, j}$ is equal to $M_{\sigma_{i}}$, see (4.6). This amounts to proving that the zeros of the functions:

$$
\theta_{l}(r)=\frac{r^{2}\left[j+\gamma_{l}(j, m)\right]-j}{r^{2}\left(1-r^{2}\right)}
$$

and

$$
\kappa_{i}(r)=\frac{\beta_{i}(j)-r^{2}\left[m-j+\beta_{i}(j)\right]}{r^{2}\left(1-r^{2}\right)}
$$

in the interval $] 0,1[$ are points where the functions change their sign. An elementary analysis of these functions show that $\theta_{l}$ is strictly increasing and $\kappa_{i}$ is strictly decreasing on ] 0,1 [ for all $l, i \geq 3$. Thus, every degeneracy instant determines a jump of the Morse index equal to its multiplicity.

The constant mean curvature torus $x_{r}^{m, j}$ has mean curvature $H_{r}^{m, j}$ given by (see [3]):

$$
H_{r}^{m, j}=\frac{m r^{2}-j}{m r \sqrt{1-r^{2}}}
$$

thus, by Proposition 3.3, part (c), the corresponding Lagrange multiplier $\lambda_{r}^{m, j}$ is:

$$
\lambda_{r}^{m, j}=\frac{m r^{2}-j}{r \sqrt{1-r^{2}}} .
$$

The derivative $\frac{\mathrm{d}}{\mathrm{d} r} \lambda_{r}^{m, j}$ is positive:

$$
\frac{\mathrm{d}}{\mathrm{d} r} \lambda_{r}^{m, j}=\frac{(m-2 j) r^{2}+j}{r^{2}\left(1-r^{2}\right)^{3 / 2}}>0,
$$

because $(m-2 j) r^{2}+j \geq \min \{j, m-j\}>0$ for $\left.r \in\right] 0,1[$.

Remark 4.5. Note that the value of $\left.r=\sqrt{\frac{j}{m}} \in\right] \sqrt{\frac{j}{m+2}}, \sqrt{\frac{j+2}{m+2}}[$, corresponding to the minimal Clifford torus, is not a degeneracy instant for the family of CMC Clifford tori.

Finally, everything is now ready for:

Proof of Theorem. The existence of CMC embeddings of the product $\mathbb{S}^{j} \times \mathbb{S}^{m-j}$ into $\mathbb{S}^{m+1}$ that accumulate at the CMC Clifford tori $x_{r_{i}}^{m, j}$ and $x_{s_{i}}^{m, j}$ and that are not congruent to any member of the CMC Clifford family corresponds exactly to the occurrence of orbit bifurcation at $r=r_{i}$ and at $r=s_{i}$ for the CMC constrained variational problem on the manifold of unparameterized embeddings of $\mathbb{S}^{j} \times \mathbb{S}^{m-j}$ into $\mathbb{S}^{m+1}$. The existence result is obtained by applying Theorem 2.7, whose assumptions are satisfied, as follows. The topological and differential structure for the manifold $\mathfrak{M}=\widetilde{\operatorname{Emb}}(M, N)$ the area 
and the volume functional $\mathcal{A}$ and $\mathcal{V}$ required in assumptions (A1)-(A5) for the CMC variational problem has been discussed in Section 3. The path of classes of CMC Clifford tori $r \mapsto\left[x_{r}^{m, j}\right]$ satisfies axioms (B1) and (B2); see (4.7) for the condition $\lambda_{r}^{\prime}>0$.

The statements in assumption (HF-A) and (HF-B) are established respectively in part (a) and (b) of Proposition 3.4. The continuity assumption (HF-B3) is deduced immediately from (3.5).

The statement of assumption (C1) is proved in Proposition 4.1. As to assumption (C2), this follows from Proposition 4.3.

The hypotheses (D1) and (D2) hold by Proposition 4.4.

Hence, by Theorem 2.7 there is orbit bifurcation at the instants $r=r_{i}$ and $s=s_{i}$, $i \in \mathbb{N}$.

As to the local rigidity of the CMC Clifford family for all other values of $r$, this follows readily as an application of Proposition 2.4 to the above setup, using the fact that, by Proposition 4.4, for all values of $r$ that do not belong to the sequences $r_{i}$ and $s_{i}$, the critical orbit $\mathrm{SO}(m+2)\left[x_{r}^{m, j}\right]$ is nondegenerate. This concludes the proof.

\section{REFERENCES}

[1] R. Abraham, J. RobBin, Transversal mappings and flows, W. A. Benjamin Inc., 1967.

[2] L. J. ALÍAs, On the stability index of minimal and constant mean curvature hypersurfaces in spheres, Rev. Unin Matemtica Argentina, vol. 47, no. 2 (2006), 39-61.

[3] L. J. Alías, A. Brasil JR., O. Perdomo, On the stability index of hypersurfaces with constant mean curvature in spheres, Proc. Am. Math. Soc. 135, no. 11 (2007), 3685-3693.

[4] L. J. Alías, A. Brasil JR., O. Perdomo, A characterization of quadric constant mean curvature hypersurfaces of spheres, J. Geom. Anal. 18, (2008), 687-703.

[5] L. J. AlíAS, P. PiCcione, On the manifold structure of the set of unparameterized embeddings with low regularity, to appear in Bull. Braz. Math. Soc. Available at http://arxiv.org/abs/1011.5075

[6] J. L. BARBosA, M. DO CARMO, Stability of hypersurfaces with constant mean curvature, Math. Z. 185 (1984), 339-353.

[7] J. L. BArbos A, M. Do CARMo, J. EschenburG, Stability of hypersurfaces with constant mean curvature in Riemannian manifolds, Math. Z. 197 (1988), 123-138.

[8] G. E. BREDon, Introduction to compact transformation groups, Pure and Applied Mathematics, Vol. 46, Academic Press, New York-London, 1972.

[9] S. Cingolani, G. Vannella, Critical groups computations on a class of Sobolev Banach spaces via Morse index, Ann. Inst. H. Poincar Anal. Non Linaire 20 (2003), no. 2, 271-292.

[10] S. Cingolani, G. Vannella, Marino-Prodi perturbation type results and Morse indices of minimax critical points for a class of functionals in Banach spaces, Ann. Mat. Pura Appl. (4) 186 (2007), no. 1, 157-185.

[11] Q. Q. CHANG, Morse theory on Banach space and its applications to partial differential equations, Chinese Ann. Math. Ser. B 4 (1983), no. 3, 381-399.

[12] K.-C. CHANG, Morse theory in nonlinear analysis. Nonlinear functional analysis and applications to differential equations, (Trieste, 1997), 60-101, World Sci. Publ., River Edge, NJ, 1998.

[13] K.-C. CHAng, Infinite-dimensional Morse theory and its applications, Sminaire de Mathmatiques Suprieures, 97. Presses de l'Universit de Montral, Montreal, QC, 1985.

[14] J.-N. CoRvellec, A. HANTOUTE, Homotopical stability of isolated critical points of continuous functionals, Set-Valued Analysis 10 (2002), 143-164.

[15] M. CRAndall, P. RaBinowitz, Bifurcation from simple eigenvalues, J. Functional Analysis 8 (1971), 321-340.

[16] E. N. DANCER, The G-invariant implicit function theorem in infinite dimension, Proc. Roy. Soc. Edinburgh Sect. A 92 (1982), no. 1-2, 13-30.

[17] E. N. DANCER, The G-invariant implicit function theorem in infinite dimension. II., Proc. Roy. Soc. Edinburgh Sect. A 102 (1986), no. 3-4, 211-220.

[18] A. DE VITA, Teoria di Morse per funzionali non regolari, Tesi di laurea in Matematica, Universit Cattolica del Sacro Cuore, Brescia, Italy, 1996.

[19] E. B. DYNKIN, The maximal subgroups of the classical groups, AMS Translations ser. 2, vol. 6 (1952), 245-278 .

[20] A. El SoufI, Applications harmoniques, immersions minimales et transformations conformes de la sphre, Compositio Math. 85 (1993), 281-298.

[21] D. Gilbarg, N. S. Trudinger, Elliptic Partial Differential Equations of Second Order, Classics in Mathematics, Springer, Reprint of the 1998 Edition, 2001. 
[22] I. Guadalupe, A. Brasil JR., J.A. Delgado, A characterization of the Clifford torus, Rend. Circ. Mat. Palermo (2) 48 (1999), 537-540.

[23] R. Hynd, S.-H. PARK, J. MCCUAn, Symmetric surfaces of constant mean curvature in $\mathbb{S}^{3}$, Pacific J. Math. 241, No. 1 (2009), 63-115.

[24] M. A. Javaloyes, A. Masiello, P. Piccione, Pseudo focal points along Lorentzian geodesics and Morse index, Adv. Nonlinear Stud. 10 (2010), no. 1, 5382.

[25] J. Jost, X. Li-Jost, X. Peng, Bifurcation of minimal surfaces in Riemannian manifolds, Trans. Amer. Math. Soc. 347 (1995), 51-62

[26] M. KILIAN, M. U. SCHMIDT, On the moduli of constant mean curvature cylinders of finite type in the 3-sphere, preprint 2008, arXiv:0712.0108v2.

[27] S. KobAYASHI, Transformation groups in differential geometry, Reprint of the 1972 edition. Classics in Mathematics. Springer-Verlag, Berlin, 1995.

[28] M. Koiso, B. PALMer, P. PiCCIONE, Bifurcation and symmetry breaking of nodoids with fixed boundary, in preparation, 2010.

[29] A. KRIEGL, P. Michor, The convenient setting for Global Analysis, M. S. M. vol. 53, Amer. Math. Soc., Providence, USA, 1997.

[30] H. B. LAwson, Lectures on minimal manifolds, vol. 1, Math. Lecture Series 9, Boston, Publish or Perish, 1980.

[31] A. Marino, G. Prodi, Metodi perturbativi nella teoria di Morse, Boll. UMI (4) 11, Suppl. fasc. 3 (1975), $1-32$.

[32] R. MazzeO, F. PACARd, Bifurcating nodoids, Contemp. Math. 314 (2002), 169-186.

[33] O. PERdomo, Low index minimal hypersurfaces of spheres, Asian J. Math. 5 (2001), 741-749.

[34] P. PICCIONE, D. V. TAUS K, On the Banach differential structure for sets of maps on non-compact domains, Nonlinear Anal. 46 (2001), no. 2, Ser. A: Theory Methods, 245-265.

[35] L. RECKE, D. PETERHOF, Abstract forced symmetry breaking and forced frequency locking of modulated waves, J. Differential Equations 144 (1998), no. 2, 233-262.

[36] W. Rossman, The first bifurcation point for Delaunay nodoids, Experiment. Math. 14 (2005), no. 3, 331342.

[37] W. Rossman, N. Sultana, Morse index of constant mean curvature tori of revolution in the 3-sphere, Illinois J. Math. 51 (2007), no. 4, 1329-1340.

[38] W. RosSman, N. Sultana, The spectra of Jacobi operators for constant mean curvature tori of revolution in the 3-sphere. Tokyo J. Math. 31 (2008), no. 1, 161-174.

[39] J. Simons, Minimal varieties in Riemannian manifolds, Ann. of Math. (2), 88 (1968), 62-105.

[40] S. SMALE, An infinite dimensional version of Sard's theorem, Amer. J. Math. 87 (1965), 861-866.

[41] J. SMOller, A. G. WASSERMAn, Bifurcation and symmetry-breaking, Invent. Math. 100 (1990), 63-95.

[42] E. H. SpAnIER, Algebraic topology, Corrected reprint. Springer-Verlag, New York-Berlin, 1981. ISBN: 0-387-90646-0

[43] K. UhlenBeCK, Morse theory on Banach manifolds, J. Functional Analysis 10 (1972), 430-445.

[44] F. Urbano, Minimal surfaces with low index in the three-dimensional sphere, Proc. Am. Math. Soc. 108 (1990), 989-992.

[45] Z. Q. WANG, Equivariant Morse theory for isolated critical orbits and its applications to nonlinear problems, Partial differential equations (Tianjin, 1986), 202-223, Lecture Notes in Math., 1306, Springer, Berlin, 1988.

[46] B. WHITE, The space of m-dimensional surfaces that are stationary for a parametric elliptic functional, Indiana Univ. Math. J. 36 (1987), 567-602.

[47] B. White, The space of minimal submanifolds for varying Riemannian metrics, Indiana Univ. Math. J. 40 (1991), 161-200.

Departamento de MATEMÁticas,

Universidad de Murcia, CAMPUS dE EsPinARdo

30100 ESPINARDO, MURCIA,

SPAIN

E-mail address: 1 jalias@um.es

DePARTAMENTo de Matemtica,

UNIVERSIDADE DE SÃo PAULO,

RUA DO MATÃO 1010 ,

CEP 05508-900, SÃo PAULO, SP, BRAZIL

E-mail address: piccione.p@gmail.com

Current address: Departamento de Matemáticas,

Universidad de Murcia, Campus de Espinardo

30100 Espinardo, Murcia,

Spain 